\title{
Imaging sensory transmission and neuronal plasticity in primary sensory neurons with a positively tuned voltage indicator
}

Yu Shin Kim ( $\nabla$ yushink@gmail.com )

University of Texas Health San Antonio https://orcid.org/0000-0002-6677-5656

\section{Yan Zhang}

University of Texas Health San Antonio

John Shannonhouse

University of Texas Health San Antonio

Ruben Gomez

University of Texas Health San Antonio

Hyeonwi Son

University of Texas Health San Antonio

Hirotake Ishida

University of Texas Health San Antonio

\section{Stephen Evans}

Stanford University

Guofeng Zhang

Stanford University

Michael Lin

Stanford University https://orcid.org/0000-0002-0492-1961

\section{Article}

Keywords: somatosensory inputs, sensory neurons, DRG, GECls, ASAP4.4-Kv

Posted Date: November 19th, 2021

DOl: https://doi.org/10.21203/rs.3.rs-852107/v1

License: (c) (1) This work is licensed under a Creative Commons Attribution 4.0 International License.

Read Full License 


\section{Abstract}

Detection of somatosensory inputs requires conversion of external stimuli into electrical signals by activation of primary sensory neurons. The mechanisms by which heterogeneous primary sensory neurons encode different somatosensory inputs remains unclear. In vivo dorsal root ganglia (DRG) imaging using genetically-encoded $\mathrm{Ca}^{2+}$ indicators (GECls) is currently the best technique for this purpose mapping neuronal function in DRG circuits by providing an unprecedented spatial and populational resolution. It permits the simultaneous imaging of $>1800$ neurons/DRG in live mice. However, this approach is not ideal given that $\mathrm{Ca}^{2+}$ is a second messenger and has inherently slow response kinetics. In contrast, genetically-encoded voltage indicators (GEVIs) have the potential to track voltage changes in multiple neurons in real time but often lack the brightness and dynamic range required for in vivo use. Here, we used soma-targeted ASAP4.4-Kv, a novel positively tuned GEVI, to dissect the temporal dynamics of noxious and non-noxious neuronal signals during mechanical, thermal, or chemical stimulation in DRG neurons of live mice. ASAP4.4-Kv is sufficiently bright and fast enough to optically characterize individual neuron coding dynamics. Notably, using ASAP4.4-Kv, we uncovered cell-to-cell electrical synchronization between adjacent DRG neurons and robust dynamic transformations in sensory coding following tissue injury. Finally, we found that a combination of GEVI and GECl imaging empowered in vivo optical studies of sensory signal processing and integration mechanisms with optimal spatiotemporal analysis.

\section{Introduction}

Dorsal root ganglia (DRG) neurons have pseudounipolar axons that project toward skin where they initially convert external stimuli such as touch, stretch, itch, hot, cold, and/or chemical stimuli into corresponding electrical signals. These electrical signals are integrated and modulated in the cell bodies of DRG located in intervertebral foramen between spinal vertebrae, and then action potentials containing somatosensory information are further propagated to the superficial laminae of dorsal spinal cord. Electrophysiologic recording has been used as a fundamental tool for measuring neuronal electrical signals for many decades, but this approach is limited by the invasiveness of the procedure, poor anatomical accessibility, the absence of physiological input during commonly used in vitro recordings, and by the difficulty of in vivo measurement due to stability issues ${ }^{1,2,3}$. Genetically-encoded $\mathrm{Ca}^{2+}$ indicators (GECls) allow for monitoring DRG neuronal firing activities, network patterns among neurons and other cell types, and sensory circuits in physiological and pathological conditions with exceptional spatial and populational resolution and limited perturbation ${ }^{4}$. However, $\mathrm{Ca}^{2+}$ indicators fail to distinguish between action potential-evoked $\mathrm{Ca}^{2+}$ influx vs. $\mathrm{Ca}^{2+}$ transients arising from internal stores and ligandgated $\mathrm{Ca}^{2+}$ channels. Furthermore, $\mathrm{Ca}^{2+}$ indicators only report suprathreshold signaling while failing to detect subthreshold membrane potential fluctuations due to slow kinetics and limited sensitivity ${ }^{2,3,5}$. As an alternative, recording DRG neuronal activity using fluorescence generated from genetically-encoded voltage indicators (GEVIs), which can follow not only fast suprathreshold voltage signals but also subthreshold fluctuations in membrane potentials, could be an excellent complementary approach to in vivo GECl imaging. 
Dynamic voltage imaging employing voltage sensing dyes and later with GEVls has been used for decades to study electrical activity in various tissues and organisms in vitro and in vivo $6,7,8,9,10$, yet no data are available pertaining to functional voltage imaging of primary sensory neurons due to the lack of appropriate tools and techniques. More recently, GEVIs have been developed that enable stable expression in mammalian cells ${ }^{11}$, allowing the use of genetic tools to achieve cell type specificity ${ }^{2,3}$. Despite the widespread use of GEVIs, only a handful have been successfully used for in vivo optical imaging to detect voltage dynamics in the living mouse brain. These include archaerhodopsin-based indicator Ace2N, paQuasAr3-s, and SomArchon ${ }^{12,13,14}$, and ASAP-family GEVIs in which a voltagesensitive domain (VSD) is linked to a circularly permuted GFP protein ${ }^{15,16,17}$. Compared to archaerhodopsin-based GEVIs, ASAP3-Kv enables accurately tracking suprathreshold and subthreshold voltage dynamics with decent signal-to-noise ratio (SNR) from deep brain regions in live mice ${ }^{15,16}$. However, ASAP3-Kv generates a reversed optical signal (a decrease in fluorescence intensity with membrane depolarization), which leads to high excitability and thus high photobleaching at resting membrane potentials. Existing positively tuned GEVIs that brighten with positive voltage changes use either VSDs from voltage sensitive phosphatases (FlicR $1^{18,19}$ and Marina ${ }^{20}$ ), or electrochromic fluorescence resonance energy transfer (eFRET) like Ace2N-mNeon ${ }^{21}$. Among these indicators, Marina exhibits largest optical responses in in vitro experiments while practical use in vivo needs more evaluations. Recent attempts have successfully developed some positively tuned ASAP4 voltage indicators that surpass currently available positively tuned voltage indicators in their fluorescence responses and SNRs ${ }^{17}$. One novel ASAP4-subfamily GEVI, ASAP4.4-Kv, which ASAP4.4 voltage indicator is attached to the Kv2.1 potassium channel to locate ASAP4.4 to the soma, combines notable properties for in vivo applications: brighten in response to membrane depolarization, high-level neuronal expression, fast kinetics, and enhanced fluorescence changes. We therefore anticipate that the properties of ASAP4.4$\mathrm{Kv}$ make it more suitable and optimal for routine and robust in vivo DRG imaging by reporting action potentials reliably and revealing subthreshold events in optical studies.

To illustrate the feasibility and utility of using ASAP4.4-Kv as an indicator in primary sensory neurons, we examined neuronal activity in the DRG of live mice. Data acquired using ASAP4.4-Kv uncovered striking cell-to-cell communication and synchronization patterns between adjacent DRG neurons following peripheral inflammation or nerve injury, which was rarely seen in naïve animals. ASAP4.4-Kv data permitted visualizing the representation of mechanical, thermal, and chemical stimuli in vivo, and enabled us to track down how these parameters transform with peripheral injury. By comparing in vivo GEVI and GECl signals, we found that GECl imaging can represent complex phenomena extending to an entire population ensemble of DRG neurons, but that images lack temporal precision and fidelity. We also found that ASAP4.4-Kv voltage imaging enabled visualization of temporal dynamics of individual DRG neurons with fast temporal resolution. We conclude that combining $\mathrm{GEVI}$ and $\mathrm{GECl}$ imaging provides an optimal approach for analyzing the complex signal processing and integration of somatosensation in various contexts. 


\section{Results}

\section{Using ASAP4.4-Kv for in vivo DRG voltage imaging}

For in vivo DRG voltage imaging, we intrathecally injected adeno-associated viruses (AAVs) encoding ASAP4.4-Kv into spinal cord to allow for expression in DRG neurons. At 5-7 weeks after injection, in vivo single photon confocal imaging experiments were performed on the right lumbar (L5) DRG, which innervates parts of right hindpaw, leg, and back of the mouse. Fluorescent signals from ASAP4.4-Kv were acquired by confocal microscopy in frame mode to capture the entire population of L5 DRG neurons. We verified transduction of ASAP4.4-Kv virus into DRG neurons by imaging ASAP4.4 fluorescence in DRG neurons. The basal ASAP4.4-Kv green fluorescence intensity was relatively low under in vivo conditions; however, inflammation in hindpaw caused by complete Freund's adjuvant (CFA) injection or chronic constriction injury ${ }^{22}$ of sciatic nerves (SNs) yielded a stronger ASAP4.4-Kv fluorescent signal (Fig. 1a and Supplementary Fig. 1a, b). The results showed that ASAP4.4-Kv can be sparsely but highly expressed in DRG neurons in vivo, and can dynamically respond to voltage in the physiological range (Supplementary Fig. 2). These are essential properties for carrying out the functional analysis at the cellular level in vivo.

We noticed that many DRG neurons showing brightly fluorescent ASAP4.4 were in close proximity to each other, more prominent in CFA and SN-CCl animals (Supplementary Fig. 1c), implying that "crossexcitation ${ }^{23 "}$ or "coupled activation ${ }^{4 "}$ may arise in proximal parts of the in vivo primary sensory neurons. This phenomenon led us to explore the utility of voltage indicators to reveal neuronal crosstalk within the peripheral nervous system of live animals.

\section{Electrical coupling synchronization between adjacent DRG neurons revealed by ASAP4.4}

DRG neurons are covered with satellite glial cells (SGCs), grouping with or without SGCs in between neighboring neurons ${ }^{24}$. Under normal circumstances, DRG neurons are loosely connected to each other or to SGCs. In contrast, extensive dye transfer and electrical coupling between adjacent neurons are often observed in many pain conditions, including inflammation in mouse hindpaw and sciatic nerve injury ${ }^{25}$, 26 , which is attributed to gap junctions present in both DRG neurons and the surrounding SGCs. As a consequence of electrical coupling by gap junction, DRG neurons exhibit coupled activation following peripheral tissue injury examined by $\mathrm{Ca}^{2+}$ imaging in DRG of live mice ${ }^{4}$.

We thus attempted to simultaneously record electrical activity between two adjacent DRG neurons using ASAP4.4. We randomly selected pairs of adjacent DRG neurons in different regions of the DRG, and performed a single-line scan at about $1.1 \mathrm{kHz}$ across the membrane regions of the two neuronal cells (Fig. 1a-c, images). We analyzed paired data sets from naïve, CFA, or SN-CCl animals by quantification of fluorescence intensity changes in scanned areas of individual adjacent cells. In naïve mice, very few DRG neurons displayed rhythmic spontaneous subthreshold voltage fluctuations, and no temporal cell-tocell coherence or synchronization of voltage signals in neuronal membranes was observed (Fig. 1a). Under the context of inflammation or nerve injury, subthreshold voltage fluctuations were readily 
detectable in vivo (Fig. 1b, c), with approximately 7-fold increase in average area under the curve (AUC) of ASAP4.4-Kv fluorescent signal intensity (Fig. 1d). Strikingly, around $6 \%$ of recording neuronal pairs exhibited spontaneous suprathreshold (spiking) activity and strong coincident voltage changes in the range of ten to hundreds of milliseconds, regardless of activity patterns (Fig. 1b, c), whereas gap junction blocker, carbenoxolone (CBX), significantly reduced cell-to-cell electrical synchronization (Fig. 1b). Our results indicate that tissue injury increased cell-to-cell connectivity and network communication between DRG neurons leading to enhanced synchronization in DRG neuronal networks, and eventually to better integration and summation of somatosensory signals. To the best of our knowledge, such electrically synchronous neuronal events between cells in the peripheral sensory system in vivo have not been previously described.

To determine whether electrically synchronous events corresponded to global neuronal activity, we included in vivo DRG $\mathrm{Ca}^{2+}$ imaging of neuronal populations using Pirt-GCaMP3 mice, in which the GECI GCaMP3 was exclusively expressed in primary sensory neurons under the control of the Pirt promoter ${ }^{27}$. Using Pirt-GCaMP3 $\mathrm{Ca}^{2+}$ imaging, we could simultaneously monitor neuronal activity of the entire population of DRG neurons ${ }^{4}$. We imaged the entire DRG at $\sim 6.4$ to $7.9 \mathrm{~s} /$ frame and found that spontaneous activity was rarely seen in naïve animals (1-3 neurons/DRG), but in the presence of inflammation or nerve injury, increased spontaneous neuronal activity was observed (>10 neurons/DRG) (Supplementary Fig. 3a). This $\mathrm{Ca}^{2+}$ activity could represent either sporadic $\mathrm{Ca}^{2+}$ oscillations or steadystate high $\mathrm{Ca}^{2+}$ (Supplementary Fig. 3b). To this point, however, no synchronized spontaneous activity was observed in GCaMP3 signals. In comparing voltage dynamics seen by ASAP4.4-Kv imaging with $\mathrm{Ca}^{2+}$ signals seen by GCaMP3 imaging, we found that ASAP4.4-Kv imaging preserved fast temporal signal information, which GCaMP3 imaging failed to convey. ASAP4.4-Kv detected numerously dynamic membrane voltage signal changes associated with inflammation or nerve injury but GCaMP3 did not (Fig. 1d and Supplementary Fig. 3c). In contrast, GCaMP3 $\mathrm{Ca}^{2+}$ signals reflected an increasing number of spontaneously activated neurons in the entire DRG after inflammation or nerve injury (Supplementary Fig. 1d,e).

\section{Noninvasive optical readout of different afferent subtypes in vivo with ASAP4.4}

Primary sensory neurons diverge in function as they express their own unique receptors and ion channels. Classically, DRG neurons are categorized into three subtypes based on somatic action potential shapes and conduction velocity; namely, myelinated $A \beta$ (large diameter and fast conducting) low threshold-

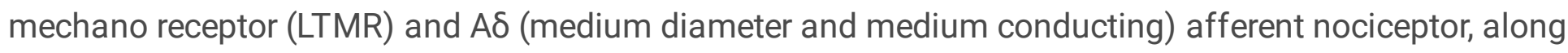
with slowly conducting small diameter unmyelinated C-type nociceptor ${ }^{28,29}$. Identification and functional characterization of different neuronal subclasses in vivo have been a challenge, thus current

investigations have mostly relied on the invasive in vitro or ex vivo electrophysiologic recordings $s^{30,31}$. As shown in Fig. 1g, we were able to visualize single action potentials by inspecting small spiking area (1-3 $\mu \mathrm{m})$ of line scan image. We found that the kinetic properties of action potentials were closely related to the sizes of DRG neurons, consistent with conventional electrophysiologic recordings ${ }^{30,32}$. This indicates 
the feasibility of in vivo voltage imaging for noninvasive optical readout of electrophysiologic features, to the point where afferent subtypes can be inferred and targeted.

\section{ASAP4.4-Kv imaging permits visualization of mechanical stimuli (non-noxious to noxious)-evoked temporal summation of fast voltage signals}

To understand how DRG neurons encode painful or non-painful mechanical stimuli, we applied stimulation of different strengths to the hindpaw, and visualized evoked ASAP4.4-Kv signals in DRG neurons. At low stimulation strength (light brush, $0.4 \mathrm{~g}$, or $2 \mathrm{~g}$ von Frey; Fig. 2), small and transient subthreshold potential changes could be observed in mechanosensitive neurons (Fig. 2a), and only a few neurons exhibited hindpaw stimulation-evoked transient $\mathrm{Ca}^{2+}$ increases in naïve animals (Fig. $2 \mathrm{~b}$ ). However, peripheral inflammation or nerve injury led to a significant increase in membrane electrical signal summation, including both subthreshold and suprathreshold voltage signals (Fig. 2a, d), but not in $\mathrm{Ca}^{2+}$ responses (Fig. 2b-d). At an intermediate stimulation strength (100 g press), high-frequency voltage dynamics were observed in neurons of naïve mice (Fig. 3a), while inflammation or nerve injury treatment produced exacerbated voltage fluctuations with larger amplitude and longer membrane depolarization (Fig. 3b-d). On the other hand, GCaMP3 $\mathrm{Ca}^{2+}$ imaging revealed increased population level activities in injured mice upon exposure to the same press stimulus (Fig. 3d, e). However, large variations in the magnitude of $\mathrm{Ca}^{2+}$ transients were found within the same DRG and across different treatment groups. Consequently, while the data were grouped, neither average amplitudes nor the mean AUCs of $\mathrm{Ca}^{2+}$ transients differed significantly between naïve or injured animals (Fig. 3d, g), despite the fact that increased activated cell numbers (Fig. 3d) and increased amplitudes of $\mathrm{Ca}^{2+}$ transients (Fig. 3f) were evident in some CFA-injured mice.

At the strongest mechanical stimulus ( $300 \mathrm{~g})$, long-lasting membrane potential fluctuations with sustained membrane depolarization were observed in DRG neurons of naïve mice (Fig, 4a), and voltage fluctuations in neuronal membranes were further aggravated by inflammation or nerve injury treatment (Fig. 4b, c and Supplementary movie 1). Similar to previous results, average $\mathrm{Ca}^{2+}$ transients differed only marginally in groups affected by inflammation or nerve injury (Fig. 4d-f), and increased numbers of activated cells were not evident (Fig. $4 \mathrm{~d}$ and Supplementary movie 7). In addition, simultaneous in vivo dual color imaging of ASAP4.4-Kv (green) and mCyRFP3 ${ }^{33}$, a cyan-excitable red fluorescent protein that can be used as a non-perturbing voltage-independent fluorescent marker as a control signal for ASAP4.4$\mathrm{Kv}$ voltage imaging, demonstrated that the pattern of evoked electrical activity was distinguishable from rhythmic physiological motions arising from respiration or heartbeat (Supplementary Fig. 4).

\section{ASAP4.4-Kv imaging reports thermal (heat or cold)-evoked voltage signals with high temporal fidelity}

It has been reported that primary sensory neurons employ different strategies to encode heat vs. cold ${ }^{34}$, 35. To discern how heat or cold is represented in vivo, we examined the ASAP4.4-Kv voltage signals from heat or cold-sensing neurons. In naïve mice, the membrane voltage dynamics during noxious heat $\left(50^{\circ} \mathrm{C}\right)$ stimulation displayed a slowly depolarizing voltage ramp that returned to baseline within $300 \mathrm{~ms}$ 
(Fig. 5a). Noxious cold $\left(0^{\circ} \mathrm{C}\right)$ stimulation, however, led to two distinctive forms of voltage activity: bursting or non-bursting (Fig. 6a). Bursting neurons displayed burst-frequency firing behaviors, whereas non-bursting neurons generated only single action potentials followed by small membrane fluctuations (Fig. 6a). Inflammation or nerve injury, in turn, resulted in augmentation of membrane voltage fluctuation and electrical amplitude in both heat- and cold-sensing neurons (Fig. 5b,c and Supplementary movie 2; Fig. 6 b,c and Supplementary movie 3). Notably, stimulation by heat or cold was represented by distinct populational signals under various pain conditions.

After CFA-induced inflammation, numerous DRG neurons were activated upon noxious heat stimulation $\left(50^{\circ} \mathrm{C}\right)$ but numbers were similar between naïve and CFA groups (Fig. $5 \mathrm{~d}$, e and Supplementary movie 8 ), while fewer neurons displayed $\mathrm{Ca}^{2+}$ activity to noxious cold $\left(0^{\circ} \mathrm{C}\right)$ compared to naïve animals (Fig. $6 \mathrm{~d}, \mathrm{e}$ ). As with the previous mechanical stimuli, heat-induced increases in $\mathrm{Ca}^{2+}$ transients were observed in some DRGs of individual CFA-treated mice (Fig. 5f), but not in grouped DRGs (Fig. 5d, g). In contrast, coldsensitive neurons displayed reduced $\mathrm{Ca}^{2+}$ transients after peripheral inflammation, both individually (Fig. 6f) and as a group (Fig. 6d, g). These results are consistent with previous reports that cold-mediated $\mathrm{Ca}^{2+}$ activity was lost in specific types of cold-sensing neurons following peripheral injury ${ }^{35}$. The discrepancy between voltage and $\mathrm{Ca}^{2+}$ signals in cold-sensing neurons suggests that, following peripheral inflammation, an individual sensory neuron still retains the ability to encode cold-specific sensory input; however, summation of the neuronal response to painful cold is suppressed by network activity in DRG.

\section{ASAP4.4-Kv imaging reveals high potassium or capsaicin-evoked strong membrane voltage fluctuations}

Finally, we used the ASAP4.4-Kv voltage sensor to examine how DRG neurons encode noxious chemical nociception. In naïve mice, direct topical application of high potassium $(50 \mathrm{mM} \mathrm{KCl})$ or capsaicin $(10$ $\mu \mathrm{M})$, a TRPV1 agonist which can initiate activity in nociceptive neurons, onto L5 DRG, resulted in $>4$-fold increase in voltage fluctuations over baseline (Fig. 7a, d). Both CFA and SN-CCI treatments significantly increased neuronal responses to $\mathrm{KCl}$ or capsaicin, with substantial increases in frequency and magnitude of dynamic membrane voltage fluctuations (Fig. 7b-d and Supplementary movie 5, 6). When the same chemical treatments were performed on Pirt-GCaMP3 mice, we observed robust activation of a large population of DRG neurons within the DRG sensory ganglia (Fig. 7f, g). Topical application of capsaicin resulted in DRG neuronal activation primarily in the small and medium diameter neurons within all populations of DRG neurons imaged (Fig. $7 \mathrm{~g}$ ). Small and medium diameter neurons are nociceptors that

typically express TRPV1 receptors. On average, the $\mathrm{Ca}^{2+}$ transients in activated neurons from injured mice were significantly higher than those from naïve animals (Fig. 7e). Compared to physical stimulation, direct chemical administration onto DRG neurons produced near-maximal $\mathrm{Ca}^{2+}$ transients and responses in most DRG neurons in vivo. These findings lead us to conclude that neuronal hypersensitivity is a common consequence of peripheral injury.

\section{Discussion}


GEVIs have been successfully used in analysis of brain regions in vivo in awake behaving mice ${ }^{12,13,14,15}$, 16,17 , and have encouraged neuroscientists to explore and unlock the full potential of the technological advances. Our current study reports the use of an improved ASAP-family GEVI, ASAP4.4-Kv, to track both spontaneous and evoked voltage activity of mouse primary sensory neurons in vivo. The ASAP4.4-Kv voltage sensor allows direct visualization of distinct temporal features of neuronal dynamics, subcellular voltage dynamics, plasticity induction, and neuronal coding in DRG, the analysis of which have been largely inaccessible and technically challenging in live animals. The ASAP4.4-Kv voltage sensor provides the means for understanding how primary sensory neurons, especially DRG neurons, function or fail to function (changes in dynamics, status, and/or pattern) at any given time under physiological and pathological conditions. Particularly attractive for DRG voltage imaging is the presented ASAP4-Kv with fast kinetics enables optical detection of single action potentials from individual DRG neurons, which allows noninvasive identification of somatosensory neuron subtypes in vivo without the aid of conventional invasive electrophysiological recordings.

\section{GEVI imaging as a powerful tool complementary to GECl imaging}

ASAP3-Kv, a previous ASAP family GEVI with desirable responsivity and SNR for in vivo use, has a negative slope relationship between voltage and fluorescence ${ }^{15}, 16$. But, new ASAP4.4-Kv produces a depolarization-dependent increase in fluorescence intensity ${ }^{17}$, as do most GCaMP GECls ${ }^{4,27}$. This new feature, combined with somatic targeting and optimal brightness, greatly improves the utility of the GEVI ASAP4.4-Kv for in vivo recording of neuronal electrical signals and activity. In our in vivo DRG voltage sensor imaging studies, we could visualize sparse signals from the membrane surface of neuronal soma expressing fluorescent ASAP4.4-Kv with imaging depths of $<20 \mu \mathrm{m}$ below the meninges membrane. We demonstrated superiority of in vivo GEVI recording for resolving long-standing debates over the temporal attributes of neuronal coding by direct comparison with in vivo $\mathrm{GECl}$ imaging modalities previously developed by our lab ${ }^{4}$. Compared to GECls, the main advantage of GEVIs is their ability to examine nonspiking (subthreshold) and/or spiking (action potential) electrical activity, because subthreshold membrane potential fluctuation and oscillation do not greatly affect internal $\mathrm{Ca}^{2+}$ levels or dynamics. ASAP4.4-Kv is able to identify non-spiking subthreshold voltage fluctuation events in DRG neurons with duration times in the millisecond range, an order of magnitude faster than the signal integration time of the $\mathrm{Ca}^{2+}$ indicator GCaMP3 or other advanced GCaMPs.

Unexpectedly, spontaneous neuronal dynamics revealed by ASAP4.4-Kv exhibited cell-to-cell coupled synchronous electrical events following injury that were normally indiscernible in GCaMP3 imaging across all DRG neurons. Highlighting the importance of in vivo voltage imaging, ASAP4.4-Kv imaging fully unmasked altered DRG neuronal electrical activities and signals resulting from peripheral injury at the single-cell level in their native environment. In contrast, variable $\mathrm{Ca}^{2+}$ activities could be observed in in vivo Prit-GCaMP3 $\mathrm{Ca}^{2+}$ imaging, but significant effects were diminished in comparisons of larger numbers of DRG neurons from multiple animals. Another fascinating aspect of fluorescence voltage sensors is their ability to map not only subthreshold depolarizing (excitatory) inputs but also 
hyperpolarizing (inhibitory) events that occur constantly in almost all neurons ${ }^{36}$. Previous studies have used voltage indicators to monitor membrane hyperpolarization in cultured neurons ${ }^{37}$, brain slices ${ }^{38}$, or in freely moving mice ${ }^{39}$. The hyperpolarizing voltage signals detected by ASAP4.4-Kv in our DRG recordings were evident in response to strong stimuli, and were detected as fluorescence intensity dropped to levels below the pre-stimulation baseline. In contrast, GECl imaging can only detect excitatory inputs, and lacks ability to detect signals related to inhibitory inputs. Thus, a plausible use of GEVI imaging would be to examine possible inhibitory signaling involved in controlling peripheral nociceptive or non-nociceptive transmission, or to examine excitatory or/and inhibitory signal summation and integration within sensory ganglia.

Given the heterogeneity of sensory neurons, integrated signals from large-scale DRG neurons need to be collected at high spatiotemporal resolution to establish cell-type or modality-specific coding strategies. The intrinsic slow kinetics of GECls permits mapping large numbers of neuronal assemblies in their native environments with conventional confocal microscopic approaches. Intensive studies using GECls have characterized sensory coding of heat or $\operatorname{cold}^{34,35,40,41}$, mechanical ${ }^{40,42,43}$, or chemical stimuli ${ }^{44}$ in health and disease conditions. Due to the intrinsic fast kinetics of GEVIs, simultaneous imaging of dozens, hundreds, or thousands of DRG neurons at high spatial (millimeters) and temporal (milliseconds) resolution is an extremely challenging task and limited by current technological advances. To overcome these limitations, we adapted a conventional, low cost, strong laser intensity, upright laser-scanning confocal microscope to be used as a versatile platform, allowing optical reporting of dynamic neuronal activity in DRG neurons at high spatial and temporal resolution by combining $\mathrm{GECl}$-based $\mathrm{Ca}^{2+}$ signals and GEVI-based voltage signals. With the continuous advances in voltage indicators and optical instruments, simultaneous voltage recording of enormous numbers of neurons in live intact peripheral tissues will enable dissecting functional connectivity in DRG circuits and mapping neuronal coding strategies with better high-throughput and greater accuracy.

\section{Future Outlook}

We previously described the phenomenon of coupled neuronal activation within DRG in mouse models of inflammatory or neuropathic pain, which is attributed to an injury-induced increase of gap juntions ${ }^{4}$. Here, our in vivo voltage imaging results provide evidence of gap-junction- mediated electrically synchronous neuronal activity between DRG neurons, further supporting increased neuronal 'cross talk' by gap junctions as an underlying mechanism in the development of hyperalgesia and allodynia. In our ongoing work, we aim to elucidate the pathway and mechanism of neuron-to-neuron transmission.

Our current implementation combining both GEVI and GECl imaging should allow detailed investigations of the relationship between suprathreshold somatic voltage signals and the corresponding $\mathrm{Ca}^{2+}$ dynamics at single-cell-, population-, or modality-specific levels. Simultaneous sub-millisecond voltage and $\mathrm{Ca}^{2+}$ imaging using a voltage-sensitive dye and $\mathrm{GECl}$ has been performed on Purkinje neurons in awake animals, and has demonstrated high spatiotemporal variations of suprathreshold voltage signals 
and $\mathrm{Ca}^{2+}$ transients between dendritic segments ${ }^{45}$. For in vivo primary sensory neuron studies, dual GEVI and GECI neuronal labeling with different fluorescence spectra will help in the analysis of correlation or integration between suprathreshold voltage signals and the resulting $\mathrm{Ca}^{2+}$ transients at high spatiotemporal resolution.

\section{Limitations Of This Study}

As a latest ASAP-family sensor, positively tuned ASAP4.4-Kv with optimal fluorescence response and SNR enables identifying and tracking each suprathreshold voltage spike during the designated time in our in vivo DRG recording. In two-photon optical imaging in deep layers of in vivo brain, a previously used ASAPfamily GEVI, ASAP3, reliably detected single spikes and resolved spikes in bursts, with appreciable optical spike amplitudes ${ }^{15}$. However, in our in vivo DRG studies, each optical trace displayed in response to different stimuli is a spatial average of voltage signals from the entire line scan encompassing surface region of $16 \mu \mathrm{m} \times 0.5 \mu \mathrm{m}$. Spatial averaging can greatly improve the SNR in optical measurements and captures more dynamic signals occurring on the cell membrane, while failing in resolving closely spaced spikes. A few spikes were detectable from a close examination of spiking activity in a small recording region, from their characteristics we were able to identify specific cell types of primary sensory neurons and their physiological firing patterns in intact living tissues.

Another limitation of our study was the use of viruses to transduce the ASAP4.4-Kv gene into DRG neurons. Viral vectors can be used in a variety of species and for cell type-specific expression, while gene delivery via virus injection can lead to uneven expression strength related to site of injection, and a limited time window of acceptable expression level ${ }^{5,46,47}$. Transgenic animals should overcome these limitations as demonstrated by our studies using the Pirt promotor to selectively drive strong GCaMP3 expression in DRG neurons ${ }^{4}$. Currently, transgenic mouse lines expressing the GEVI have been reported for in vivo studies in olfactory cells ${ }^{48}$. Future engineering efforts could focus on transgenic GEVI mouse lines for selective expression in targeted tissues, which promise spatially homogeneous transgene expression and long-term time windows for GEVI imaging.

In conclusion, ASAP4.4-Kv voltage imaging opens new avenues to explore the basic principles of DRG neuron coding, and of the cellular basis for perceptual changes in somatosensation by providing high temporal resolution of individual neurons. The combination of GEVI and GECl imaging allows a more temporally and spatially precise characterization of the neuronal coding and integration strategies in the peripheral somatosensory system.

\section{Methods}

\section{Animal models}

All experiments were performed in accordance with a protocol approved by the Institutional Animal Care and Use Committee at University of Texas Health Science Center at San Antonio (UTHSA). C57BL/6J 
mice (body weight 20-30 g) were obtained and bred in-house. Animals were group housed unless otherwise noted, provided with food and water ad libitum, and kept on a $14 / 10$ light/dark cycle at $23^{\circ} \mathrm{C}$. To generate CFA inflammatory injury mice, we made a 1:1 mixture of complete Freund's adjuvant (CFA): saline, and injected $50 \mu \mathrm{L}$ subcutaneously into the glabrous skin of the hindpaw. In vivo imaging was performed 1-3 days following CFA injection. To generate sciatic nerve (SN) chronic constriction injury ${ }^{22}$, mice were anesthetized by intraperitoneal (i.p.) injection of ketamine/xylazine $(0.1 / 0.015 \mathrm{mg} / \mathrm{g}$ body weight). SN was exposed mid-thigh by a small incision and separated from surrounding tissue. Ligatures were loosely tied using 3-0 silk thread around SN. The incision was closed using sutures, and mice were used for in vivo imaging 7-10 days later.

Pirt-GCaMP3 mice were generated and described as done in a previous study ${ }^{4,25}$. Briefly, transgenic animals were generated by targeted homologous recombination to replace the entire coding region of the Pirt gene with the GCaMP3 sequence in frame with the Pirt promoter.

\section{ASAP4.4-Kv virus delivery}

AAV8-hSyn-ASAP4.4-Kv and AAV8-hSyn-ASAP4.4-Kv-mCyRFP3.WPRE ${ }^{17}$ were generated by the Stanford Viral Core. For the experiments in cell culture, male or female 2 to 3 weeks-old mice were used for intrathecal delivery of the virus to DRG neurons. For in vivo imaging, 2 to 4 months old mice from both sexes were used for intrathecal delivery of virus to peripheral neurons. For intrathecal delivery, mice from both sexes were first anesthetized with isoflurane, shaved and disinfected. The AAVs were diluted in sterile, isotonic saline. A volume of $30 \mu$ containing $2 \times 10^{12}$ virus particles $/ \mathrm{ml}$ was injected intrathecally (i.t.) by direct lumbar puncture using a 281/2-gauge needle and insulin syringe (Becton Dickinson, Franklin Lakes, $\mathrm{NJ}$ ). A reflexive flick of the tail indicated proper needle entry location for intrathecal injection. Following the injection, the animals were returned to recovery cages where they remained for 5-7 weeks until imagining or electrophysiology experiments were performed.

\section{DRG exposure surgery}

DRG exposure surgery was carried out as previously described ${ }^{4}$. Briefly, mice were anesthetized with i.p. injection of ketamine/xylazine $(0.1 / 0.015 \mathrm{mg} / \mathrm{g}$ body weight). Mice were kept on a heating pad to maintain body temperature at $37 \pm 0.5^{\circ} \mathrm{C}$, which was monitored by a rectal probe. Their backs were shaved, and ophthalmic ointment was applied to their eyes to prevent drying. The transverse processes of lumbar L5 were exposed, and the surface aspect of the bone covering the DRG was carefully removed to expose the underlying DRG without damaging the DRG or spinal cord. Bleeding was gently stopped using styptic cotton or gel foam. 


\section{In vivo imaging}

For in vivo imaging of the whole L5 DRG, mice were placed on a custom-built tilted stage and their spines were secured with custom-built clamps to minimize movement due to breathing and heartbeat. The stage was affixed under a LSM 800 confocal laser-scanning microscope (Carl Zeiss, Inc) equipped with upright $5 \times, 10 x$, and $40 x$ objectives. The isoflurane-anesthetized animals $\left(1 \%-2 \%\right.$, vol/vol in $\left.100 \% \mathrm{O}_{2}\right)$ were maintained at $37 \pm 0.5^{\circ} \mathrm{C}$ by a heating pad during the imaging process. Z-stack imaging, which can cover the entire L5 DRG, was typically acquired at eight to ten frames using a 10× C Epiplan-Apochromat objective (0.4-NA, 5.4-mm free working distance, Carl Zeiss) at typically $512 \times 512$ pixel resolution with lasers tuned at $488 \mathrm{~nm}$ and at $561 \mathrm{~nm}$ and emission at 500-550 nm for green and $620-700 \mathrm{~nm}$ for red fluorescence. DRG neurons were at the focal plane, and imaging was monitored during the activation of DRG neuron cell bodies by peripheral hindpaw stimuli.

For the recording of ASAP4.4-Kv fluorescent signals, z-stack imaging was first performed to localize the individual spiking neurons which responded to peripheral hindpaw sensory stimuli. Next, bidirectional line scanning along neuronal cell bodies was performed on the chosen cell to achieve fast ASAP4.4 imaging at $1.1 \mathrm{kHz}$. After adjusting focus depths to avoid artifacts of membrane motion, around 7700 lines (7 s) at $<1 \mathrm{~ms}$ per line, $128 \times 4$ pixels in image size, and $0.12 \mu \mathrm{m}$ in pixel size were acquired per stimulus. For reporting cell-to-cell communication, around 5500 lines ( $5 \mathrm{~s}$ ) at $<1 \mathrm{~ms}$ per line were imaged at $1024 \times 1$ pixels in image size and $0.02 \mu \mathrm{m}$ in pixel size. Fluorescent signals of each line were integrated to produce a movie file of fluorescent trace over time. For ASAP4.4 signal analysis, the first 500-2000 lines were typically discarded due to photobleaching, and fluorescent traces with strong motion artifacts were also excluded in the analysis.

\section{Stimulus delivery during imaging experiments}

Mechanical or thermal stimuli were applied on the ipsilateral hindpaw in the following order: brush, $0.4 \mathrm{~g}$ von Frey, $2 \mathrm{~g}$ von Frey, $100 \mathrm{~g}$ press, $300 \mathrm{~g}$ press, heat $\left(50^{\circ} \mathrm{C}\right)$, or cold $\left(0^{\circ} \mathrm{C}\right)$. Paw stimuli with $100 \mathrm{~g}$ or $300 \mathrm{~g}$ press force were delivered using a rodent pincher analgesia meter, and press force was controlled manually by the experimenter. For the ASAP4.4 imaging, the duration of the external stimuli was 4-5 s after 2-3 s of baseline imaging. For the GCaMP3 imaging, a time series of a total of 20 cycles was performed for each stimulus. The first 5 cycles (40-50 s) of z-stack images were captured for baseline activity determination, and images for another 5 cycles (40-50 s) were taken when paw or DRG stimuli were applied. Images were continuously taken for a total of 20 cycles. At the end of experiments, $20 \mu \mathrm{l}$ of $50 \mathrm{mM} \mathrm{KCl}$ or $10 \mu \mathrm{M}$ capsaicin was applied dropwise to the L5 DRG following 5 cycles of baseline imaging. After an incubation period of 1-2 cycles (5-10 s), $\mathrm{KCl}$ or capsaicin solution was removed by Kimwipe tissue, and then an additional 5 cycles of images were captured. Each stimulus was separated by an interval of 3-10 mins resting time for mice to avoid sensitization of neurons. 


\section{DRG culture}

ASAP4.4-transduced mice of either sex (6-7 weeks old, 4 weeks after intrathecal delivery of AAV8-hSynASAP4.4-Kv virus) were anesthetized with isoflurane, and euthanized by decapitation. DRG were removed bilaterally at L3-L5 and incubated in collagenase (Worthington) and dispase (Sigma-Aldrich) for 40 min at $37^{\circ} \mathrm{C}$ with gentle agitation every $10 \mathrm{~min}$. The dissected DRG neurons were then triturated, centrifuged, and resuspended in Dulbecco's Modified Eagle Medium (DMEM, Gibco, Grand Island, NY) supplemented with $10 \%$ fetal bovine serum (FBS, Gibco), $100 \mathrm{ng} / \mathrm{ml}$ nerve growth factor (NGF, Harlan, Indianapolis, IN), $1 \%$ penicillin/streptomycin (Gibco), and 1\% L-glutamine (Gibco), and then placed on coverslips coated with poly-D-lysine and laminin (Corning, Corning, NY). Cultures were maintained at $37^{\circ} \mathrm{C}, 5 \% \mathrm{CO}_{2}$ for $24 \mathrm{hr}$ prior to electrophysiologic recordings.

\section{In vitro electrophysiologic recording and green fluorescence imaging with ASAP4.4}

Whole-cell patch-clamp recordings were performed under voltage-clamp mode using an Muticlamp 700B amplifier and pClamp11 software (Molecular Devices), and data were digitized using an Axon Instruments Digitizer. Pipette membrane capacitance was compensated, and currents were sampled at 10 kHz. Glass pipettes (3-4 M $\mathrm{M}$ resistance, World Precision Instruments (Sarasota, FL)) were filled with an intracellular solution containing the following: $140 \mathrm{mM} \mathrm{K}$-gluconate, $5 \mathrm{mM} \mathrm{KCl}, 10 \mathrm{mM} \mathrm{HEPES}, 5 \mathrm{mM}$ $\mathrm{MgCl}_{2}, 4 \mathrm{mM}$ Mg-ATP, $0.3 \mathrm{mM} \mathrm{Na-GTP,} \mathrm{pH} \mathrm{7.2.} \mathrm{The} \mathrm{coverslips} \mathrm{containing} \mathrm{DRG} \mathrm{neurons} \mathrm{were} \mathrm{transferred} \mathrm{to}$ the recording chamber and continuously perfused with recording solution containing: $125 \mathrm{mM} \mathrm{NaCl}$, $2.5 \mathrm{mM} \mathrm{KCl}, 2 \mathrm{mM} \mathrm{CaCl}_{2}, 1 \mathrm{mM} \mathrm{MgCl}_{2}, 1.5 \mathrm{mM} \mathrm{NaH}_{2} \mathrm{PO}_{4}, 15 \mathrm{mM} \mathrm{NaHCO}_{3}$ and $10 \mathrm{mM}$ D-glucose (pH 7.4), and bubbled with $5 \% \mathrm{CO}_{2} / 95 \% \mathrm{O}_{2}$.

Fluorescence traces were acquired with cells using whole-cell voltage-clamp mode. Step voltage was applied to change the membrane potential from a holding voltage of $-70 \mathrm{mV}$ to command voltages at $-100,-40,+30$, or $+100 \mathrm{mV}$ in a series of subsequent steps for $0.5-1 \mathrm{~s}$. ASAP4.4-Kv expressing DRG neurons were imaged on an upright Zeiss Examiner.A1 microscope fitted with a 40x water-immersion objective (0.75-NA, 2.1-mm free working distance, Carl Zeiss) and with an Axiocam 705 color camera (Carl Zeiss). Images were sampled at $5 \mathrm{~Hz}$.

\section{In vivo imaging data analysis}

To analyze confocal line-scan imaging of ASAP4.4, fluorescence imaging data were extracted from raw image data, and time-dependent fluorescence traces for each neuron were revealed using Mean ROI function in Zen blue software. Because presentation of peripheral stimuli evoked spatially differentiated, large optical signals that were distinguishable from the stimulus-independent component, we averaged the first $1-2 \mathrm{~s}$ before the stimulus onset and designated that as the baseline fluorescence $\left(F_{0}\right)$. Baseline- 
normalized amplitudes in the region of interest $(\mathrm{ROI})$ over time were expressed as $\left(\mathrm{F}-\mathrm{F}_{0}\right) / \mathrm{F}_{0} \times 100 \%$ against time. Some experiments were excluded if rundown exceeded $30 \%$.

For GCaMP3 imaging data analysis, individual responding neurons were verified by visual examination and confirmed when the fluorescent intensity of ROI during stimulus was $15 \%$ higher than baseline signals using the Mean ROI function in Zen blue software. Time series recorded fluorescence changes were exported to Excel, and were analyzed using GraphPad prism. The average fluorescence intensity in the baseline period was taken as $F_{0}$, and was measured as the average pixel intensity during the first two to five frames of each imaging experiment. Relative change in fluorescence intensity was measured using the formula $\Delta F / F_{0}(\%)=\left(F-F_{0}\right) / F_{0} \times 100 \%$.

\section{Statistical methods}

Group data were expressed as mean \pm standard error of the mean (S.E.M.). Student's unpaired t-tests, Mann-Whitney U-tests, one-way ANOVA with a post-hoc Dunnett's t-test, or Kruskal-Wallis test, as appropriate, were employed for comparisons. A two-tailed $p$ value $<0.05$ was considered statistically significant for all analyses. All statistical tests are indicated in figure legends.

\section{Declarations}

\section{Acknowledgments}

Funding was obtained from the National Institutes of Health Grant R01DE026677 (Y. S. K), UTHSA Startup from University of Texas system (Y. S. K) and Rising STAR Award from University of Texas system (Y. S. K).

\section{Author contributions}

Y.Z. and Y.S.K contributed to study design with assistance from J.S., R.G., H.S., H.I., and M.L.. M.C. and M.L. developed the predecessor to ASAP4.4 voltage sensor. D.S. finalized and made the ASAP4.4 sensor. G.Z. cloned the ASAP4.4 viral constructs. M.C. made the ASAP4.4 virus. Y.S.K. contributed to data interpretation and manuscript revision. Y.Z. conceived the project and performed all experiments except where noted, and drafted the paper. R.G. and J.S. maintained, set up mating, took care of mice, and performed genotyping. R.G. assisted with GCaMP imaging work. Y.S.K. supervised all aspects of the project and wrote the paper.

\section{Competing interests}


Authors declare that they have no competing interests.

\section{Data availability}

All data are available in the main text or the supplementary materials.

\section{References}

1. Anderson $M$, Zheng Q, Dong X. Investigation of Pain Mechanisms by Calcium Imaging Approaches. Neurosci Bull 34, 194-199 (2018).

2. Antic SD, Empson RM, Knöpfel T. Voltage imaging to understand connections and functions of neuronal circuits. J Neurophysio/ 116, 135-152 (2016).

3. Lin MZ, Schnitzer MJ. Genetically encoded indicators of neuronal activity. Nat Neurosci 19, 11421153 (2016).

4. Kim YS, et al. Coupled Activation of Primary Sensory Neurons Contributes to Chronic Pain. Neuron 91, 1085-1096 (2016).

5. Chen TW, et al. Ultrasensitive fluorescent proteins for imaging neuronal activity. Nature 499, 295-300 (2013).

6. Cohen LB, et al. Changes in axon fluorescence during activity: molecular probes of membrane potential. J Membr Biol 19, 1-36 (1974).

7. Fujii S, Hirota A, Kamino K. Optical signals from early embryonic chick heart stained with potential sensitive dyes: evidence for electrical activity. J Physiol 304, 503-518 (1980).

8. Loew LM, Cohen LB, Salzberg BM, Obaid AL, Bezanilla F. Charge-shift probes of membrane potential. Characterization of aminostyrylpyridinium dyes on the squid giant axon. Biophys $J$ 47, 71-77 (1985).

9. Fukunishi K, Murai N, Uno H. Dynamic characteristics of the auditory cortex of guinea pigs observed with multichannel optical recording. Biol Cybern 67, 501-509 (1992).

10. Cha $\mathrm{MH}$, et al. Modification of cortical excitability in neuropathic rats: a voltage-sensitive dye study. Neurosci Lett 464, 117-121 (2009).

11. Dimitrov D, et al. Engineering and characterization of an enhanced fluorescent protein voltage sensor. PLoS One 2, e440 (2007).

12. Gong $Y$, et al. High-speed recording of neural spikes in awake mice and flies with a fluorescent voltage sensor. Science 350, 1361-1366 (2015).

13. Adam $Y$, et al. Voltage imaging and optogenetics reveal behaviour-dependent changes in hippocampal dynamics. Nature 569, 413-417 (2019).

14. Piatkevich KD, et al. Population imaging of neural activity in awake behaving mice. Nature $574,413-$ 417 (2019). 
15. Villette V, et al. Ultrafast Two-Photon Imaging of a High-Gain Voltage Indicator in Awake Behaving Mice. Cell 179, 1590-1608 e1523 (2019).

16. Wu J, et al. Kilohertz two-photon fluorescence microscopy imaging of neural activity in vivo. Nat Methods 17, 287-290 (2020).

17. Evans SW, et al. Simultaneous imaging of calcium and electrical activity in the brain with a positively tuned voltage indicator. (2021).

18. Abdelfattah AS, et al. A Bright and Fast Red Fluorescent Protein Voltage Indicator That Reports Neuronal Activity in Organotypic Brain Slices. J Neurosci 36, 2458-2472 (2016).

19. Milosevic MM, Jang J, McKimm EJ, Zhu MH, Antic SD. In Vitro Testing of Voltage Indicators: Archon1, ArcLightD, ASAP1, ASAP2s, ASAP3b, Bongwoori-Pos6, BeRST1, FlicR1, and Chi-VSFPButterfly. eNeuro 7, (2020).

20. Platisa J, Vasan G, Yang A, Pieribone VA. Directed Evolution of Key Residues in Fluorescent Protein Inverses the Polarity of Voltage Sensitivity in the Genetically Encoded Indicator ArcLight. ACS Chem Neurosci 8, 513-523 (2017).

21. Abdelfattah AS, et al. A general approach to engineer positive-going eFRET voltage indicators. Nat Commun 11, 3444 (2020).

22. Blum E, Procacci P, Conte V, Hanani M. Systemic inflammation alters satellite glial cell function and structure. A possible contribution to pain. Neuroscience 274, 209-217 (2014).

23. Devor M, Wall PD. Cross-excitation in dorsal root ganglia of nerve-injured and intact rats. $J$ Neurophysiol 64, 1733-1746 (1990).

24. Hanani M, Spray DC. Emerging importance of satellite glia in nervous system function and dysfunction. Nat Rev Neurosci 21, 485-498 (2020).

25. Dublin P, Hanani M. Satellite glial cells in sensory ganglia: their possible contribution to inflammatory pain. Brain Behav Immun 21, 592-598 (2007).

26. Hanani M, Huang TY, Cherkas PS, Ledda M, Pannese E. Glial cell plasticity in sensory ganglia induced by nerve damage. Neuroscience 114, 279-283 (2002).

27. Kim YS, et al. Central terminal sensitization of TRPV1 by descending serotonergic facilitation modulates chronic pain. Neuron 81, 873-887 (2014).

28. Basbaum Al, Bautista DM, Scherrer G, Julius D. Cellular and molecular mechanisms of pain. Cell 139, 267-284 (2009).

29. Lawson SN. Phenotype and function of somatic primary afferent nociceptive neurones with C-, Adelta- or Aalpha/beta-fibres. Exp Physio/ 87, 239-244 (2002).

30. Waddell PJ, Lawson SN. Electrophysiological properties of subpopulations of rat dorsal root ganglion neurons in vitro. Neuroscience 36, 811-822 (1990).

31. Koerber HR, Woodbury CJ. Comprehensive phenotyping of sensory neurons using an ex vivo somatosensory system. Physiol Behav 77, 589-594 (2002). 
32. Fang X, McMullan S, Lawson SN, Djouhri L. Electrophysiological differences between nociceptive and non-nociceptive dorsal root ganglion neurones in the rat in vivo. J Physio/ 565, 927-943 (2005).

33. Kim, B. B. et al. A red fluorescent protein with improved monomericity enables ratiometric voltage imaging with ASAP3. bioRxiv (2020).

34. Wang F, et al. Sensory Afferents Use Different Coding Strategies for Heat and Cold. Cell Rep 23, 2001-2013 (2018).

35. Yarmolinsky DA, Peng Y, Pogorzala LA, Rutlin M, Hoon MA, Zuker CS. Coding and Plasticity in the Mammalian Thermosensory System. Neuron 92, 1079-1092 (2016).

36. Knopfel T, Song C. Optical voltage imaging in neurons: moving from technology development to practical tool. Nat Rev Neurosci 20, 719-727 (2019).

37. St-Pierre F, Marshall JD, Yang Y, Gong Y, Schnitzer MJ, Lin MZ. High-fidelity optical reporting of neuronal electrical activity with an ultrafast fluorescent voltage sensor. Nat Neurosci 17, 884-889 (2014).

38. Nakajima R, Baker BJ. Mapping of excitatory and inhibitory postsynaptic potentials of neuronal populations in hippocampal slices using the GEVl, ArcLight. J Phys D Appl Phys 51, (2018).

39. Marshall JD, et al. Cell-Type-Specific Optical Recording of Membrane Voltage Dynamics in Freely Moving Mice. Cell 167, 1650-1662 e1615 (2016).

40. Chisholm KI, Khovanov N, Lopes DM, La Russa F, McMahon SB. Large Scale In Vivo Recording of Sensory Neuron Activity with GCaMP6. eNeuro 5, (2018).

41. Luiz AP, et al. Cold sensing by NaV1.8-positive and NaV1.8-negative sensory neurons. Proc Natl Acad Sci U S A 116, 3811-3816 (2019).

42. Ghitani N, et al. Specialized Mechanosensory Nociceptors Mediating Rapid Responses to Hair Pull. Neuron 95, 944-954 e944 (2017).

43. Kucharczyk MW, Chisholm KI, Denk F, Dickenson AH, Bannister K, McMahon SB. The impact of bone cancer on the peripheral encoding of mechanical pressure stimuli. Pain 161, 1894-1905 (2020).

44. Leijon SCM, Neves AF, Breza JM, Simon SA, Chaudhari N, Roper SD. Oral thermosensing by murine trigeminal neurons: modulation by capsaicin, menthol and mustard oil. J Physio/ 597, 2045-2061 (2019).

45. Roome CJ, Kuhn B. Simultaneous dendritic voltage and calcium imaging and somatic recording from Purkinje neurons in awake mice. Nat Commun 9, 3388 (2018).

46. Tian L, et al. Imaging neural activity in worms, flies and mice with improved GCaMP calcium indicators. Nat Methods 6, 875-881 (2009).

47. Dana $\mathrm{H}$, et al. Sensitive red protein calcium indicators for imaging neural activity. Elife 5, (2016).

48. Platisa, J. et al. Voltage imaging using transgenic mouse lines expressing the GEVI ArcLight in two olfactory cell types. bioRxiv (2020).

\section{Supplementary Information}


Supplementary Figure 1. Representative images of in vivo entire L5 DRG neurons expressing ASAP4.4.

Supplementary Figure 2. ASAP4.4 is well suited for optical imaging responses across the physiological voltage range in DRG neurons.

Supplementary Figure 3. Spontaneous $\mathrm{Ca}^{2+}$ activity in DRG neurons measured optically with geneticallyencoded $\mathrm{Ca}^{2+}$ indicator using Pirt-GCaMP3 mice.

Supplementary Figure 4. ASAP4.4 optical signal recording is not affected by motion.

Supplementary Movie 1. Representative in vivo optical ASAP4.4 recording of a L5 DRG neuron from SN$\mathrm{CCl}$ mouse in response to $300 \mathrm{~g}$ press applied to hindpaw.

Supplementary Movie 2. Representative noxious heat $\left(50^{\circ} \mathrm{C}\right)$-induced dynamic voltage imaging of a $\mathrm{L} 5$ DRG neuron from SN-CCl mouse.

Supplementary Movie 3. Representative noxious cold $\left(0^{\circ} \mathrm{C}\right)$-induced dynamic voltage imaging of a L5 DRG neuron from CFA-injected mouse.

Supplementary Movie 4. Representative in vivo optical ASAP4.4 recording of a L5 DRG neuron from SN$\mathrm{CCl}$ mouse before application of chemical stimuli onto DRG neuron.

Supplementary Movie 5. Representative in vivo optical ASAP4.4 recording of a L5 DRG neuron from SN$\mathrm{CCl}$ mouse in response to topical application of $\mathrm{KCl}(50 \mathrm{mM})$.

Supplementary Movie 6. Representative in vivo optical ASAP4.4 recording of a L5 DRG neuron from SN$\mathrm{CCl}$ mouse in response to topical application of capsaicin $(10 \mu \mathrm{M})$. 
Supplementary Movie 7. Representative in vivo entire L5 DRG Pirt-GCaMP3 $\mathrm{Ca}^{2+}$ imaging from naïve and CFA-treated mice in response to $300 \mathrm{~g}$ mechanical press to hindpaw.

Supplementary Movie 8. Representative in vivo entire L5 DRG Pirt-GCaMP3 $\mathrm{Ca}^{2+}$ imaging from naïve and CFA-treated mice in response to noxious heat $\left(50^{\circ} \mathrm{C}\right)$ stimulus to hindpaw.

\section{Figures}

\section{Figure 1}

a
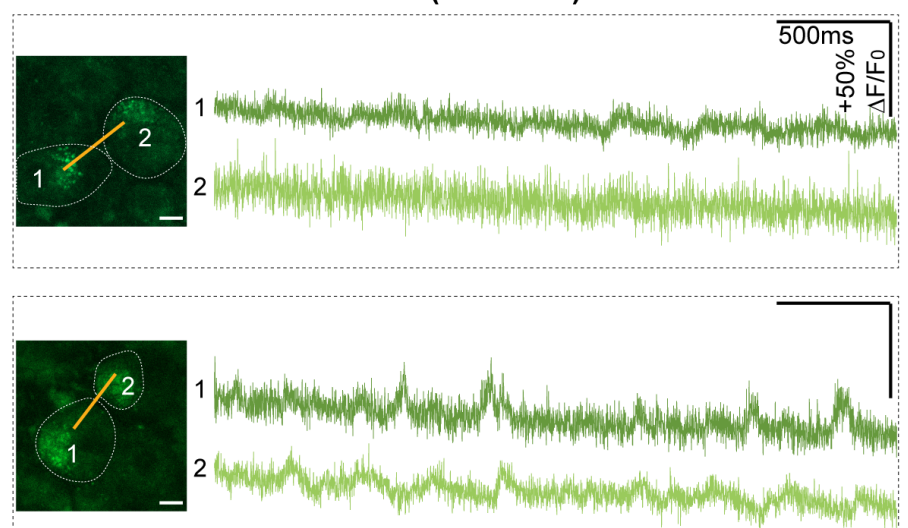

b

CFA (ASAP4.4)
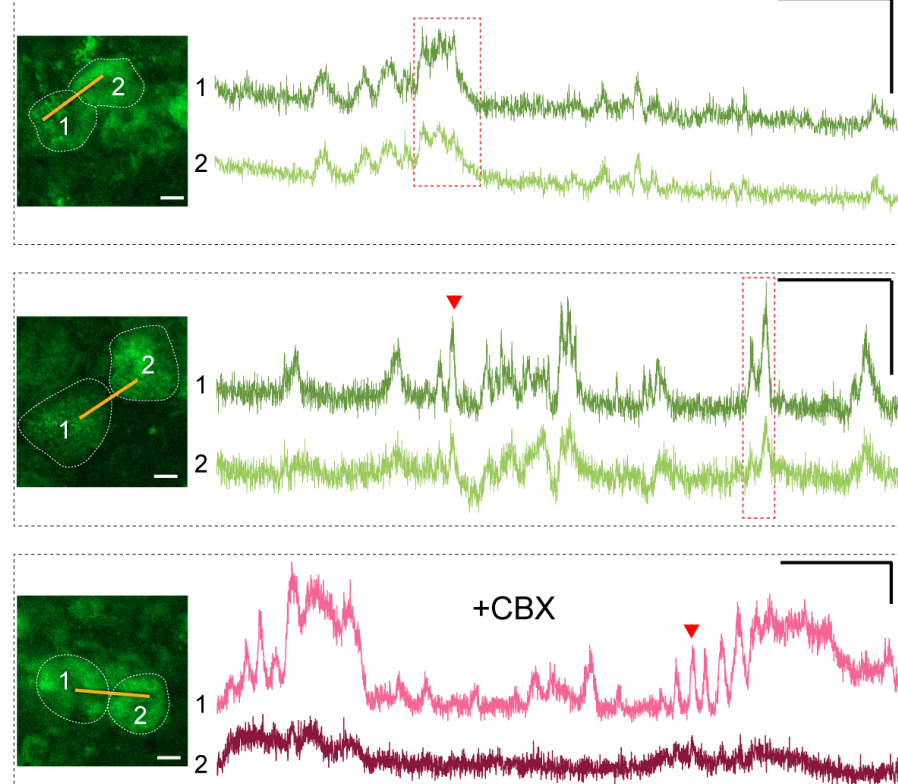

$+\mathrm{CBX}$

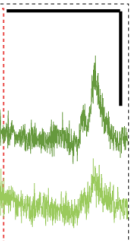

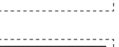

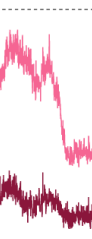

C

SN-CCI (ASAP4.4)
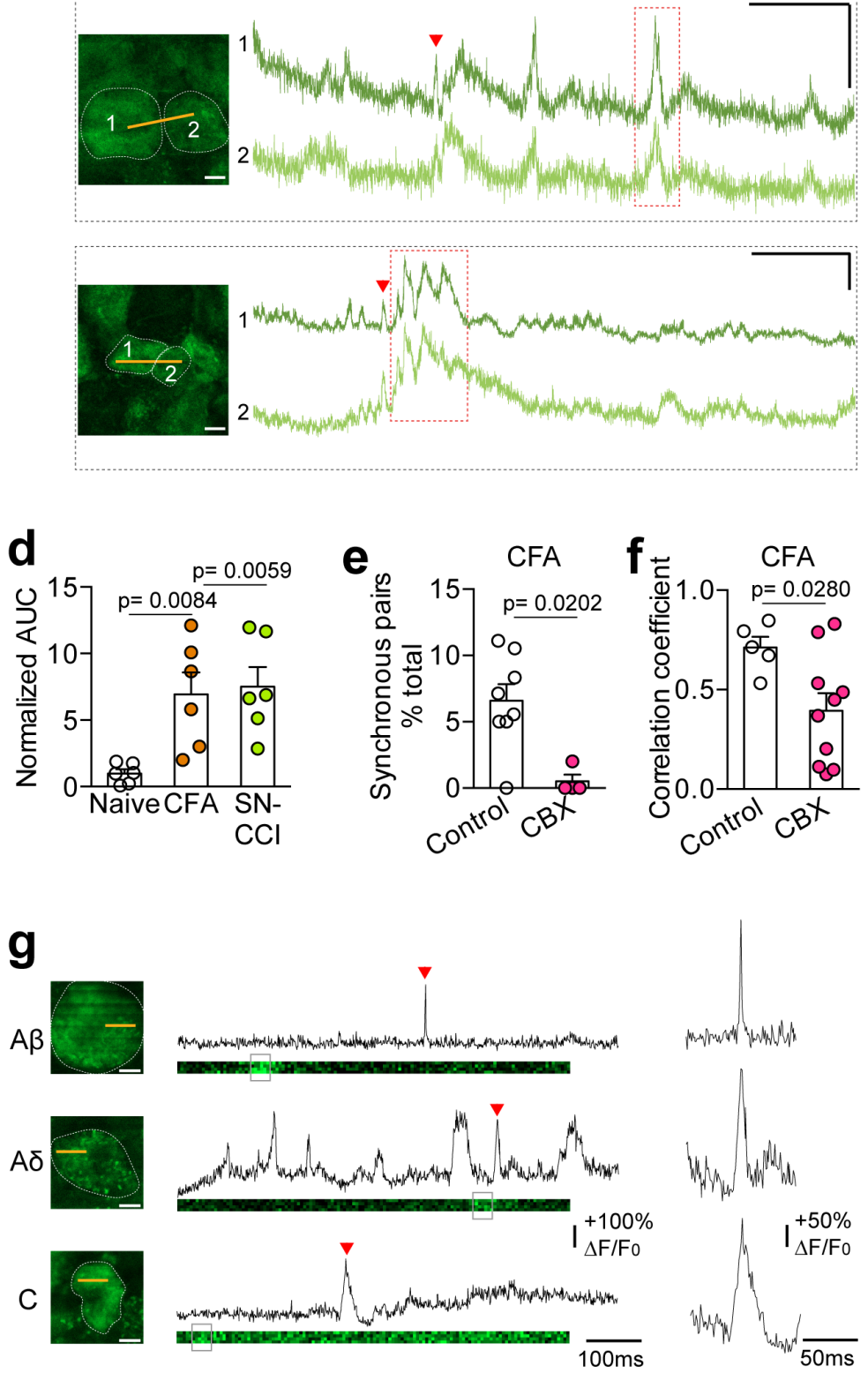


\section{Figure 1}

In vivo optical detection of electrically synchronous, spontaneous neuronal activity using ASAP4.4 voltage imaging in intact DRG neurons. a-c Paired optical recordings of two DRG somata expressing ASAP4.4 in naïve (a), CFA (b), or SN-CCI (c) animals. Yellow lines indicate $1.1 \mathrm{kHz}$ line scan regions where ASAP4.4 fluorescent signals were acquired. In (b), representative traces show electrical desynchronization between a pair of adjacent DRG neurons approximately $1 \mathrm{hr}$ after systemic gap junction blocker, CBX, injection (100 mg/kg, i.p.). The subthreshold voltage dynamics from recording neurons were gradually diminished following de-synchronization by gap junction blocker. Red arrow marks single action potential and red box indicates action potential burst. $d$ Mean area under the curve (AUC) of ASAP4.4 signals in L5 DRG neurons from naïve, CFA, or SN-CCl animals (each dot represents one neuron, $\mathrm{n}=3$ mice/group; Kruskal-Wallis test). e Quantification of synchronous pairs in control and after systemic CBX injection (Each dot represents one CFA-treated animal, Mann-Whitney U-test). $f$ Correlation coefficient was calculated from DRG neuron pairs in control and after systemic CBX injection (Each dot represents one pair of DRG neurons from a CFA-treated animal, Mann-Whitney U-test). Neuron pairs exhibit electrical correlation, whereas the correlation is dissociated by gap junction blocker. Fluorescence traces showing respiratory motion or heartbeat-like rhythmic events, or digital artifacts were not considered as electrical synchronization in the analysis. g Optical ASAP4.4 signals from DRG neurons of different size reveal various action potential shapes, in agreement with $A \beta, A \delta$ and C-type nociceptor properties. Grey box on the line scan image indicates regions of interest (ROI). Red arrow marks single action potential recorded from ROls, with expanded view on the right. Scale bar in inset image: $10 \mu \mathrm{m}$. 
a

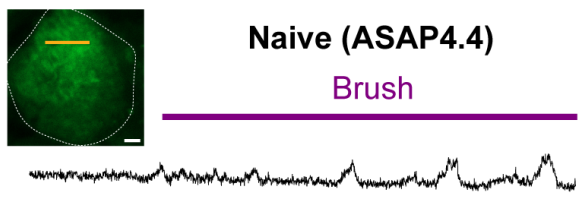

$0.4 \mathrm{~g}$ von Frey
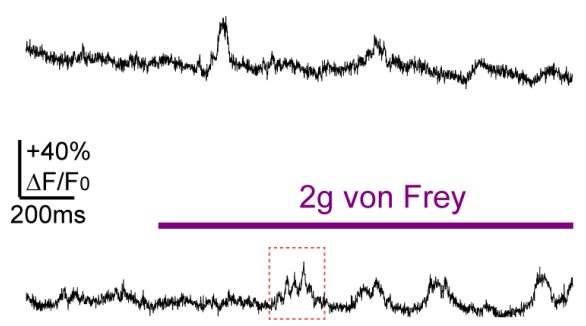

b

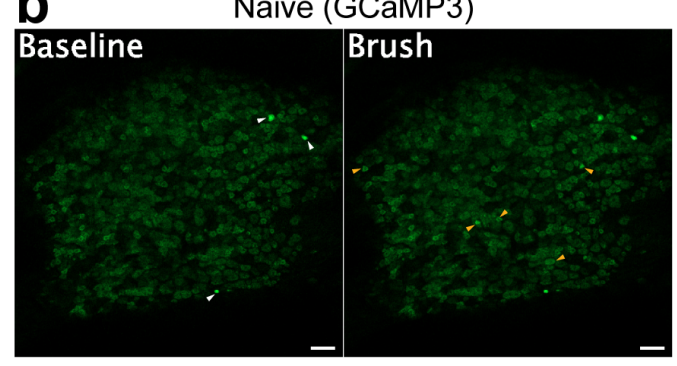

CFA (GCaMP3)

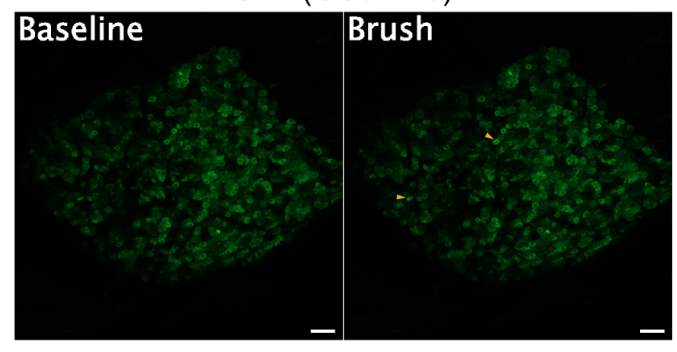

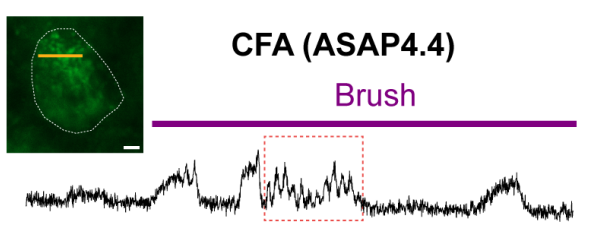

$0.4 \mathrm{~g}$ von Frey
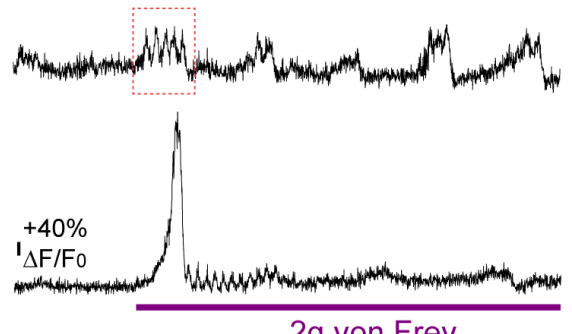

$2 \mathrm{~g}$ von Frey
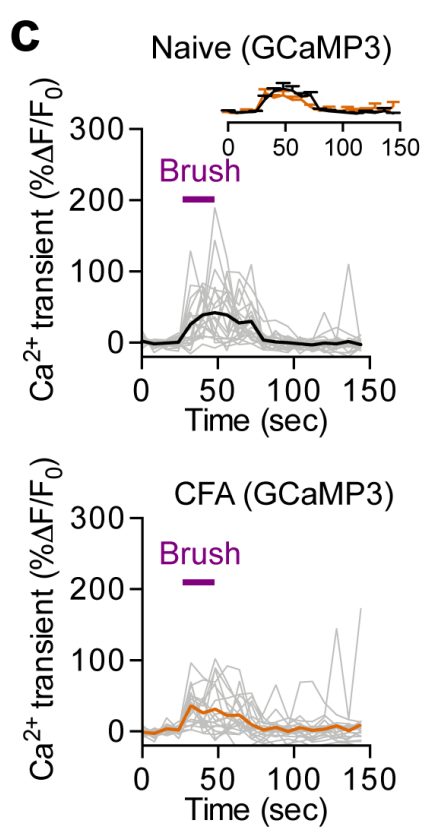

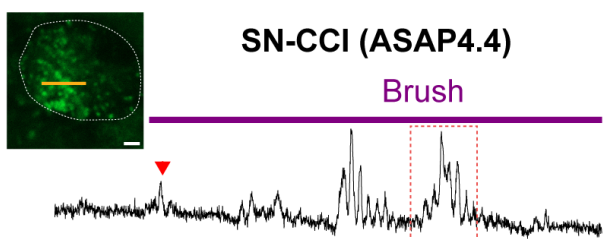

$0.4 \mathrm{~g}$ von Frey

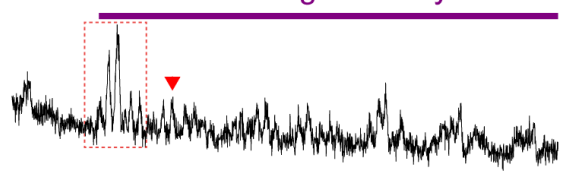

$2 \mathrm{~g}$ von Frey
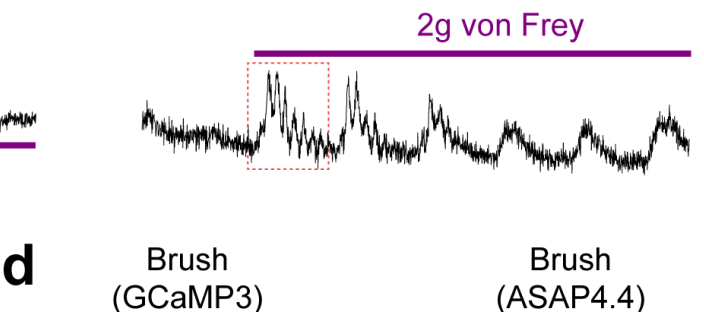

Brush

(ASAP4.4)
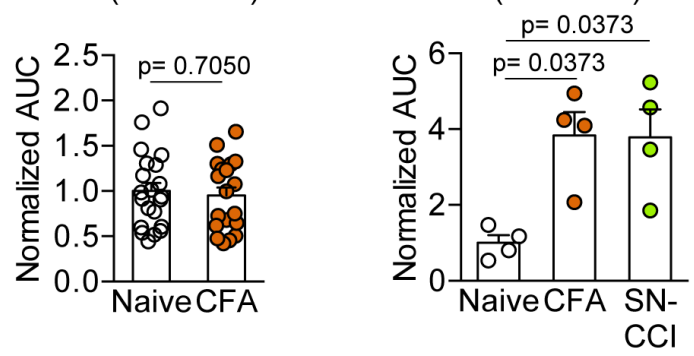

$0.4 \mathrm{~g}$ von Frey

(ASAP4.4)

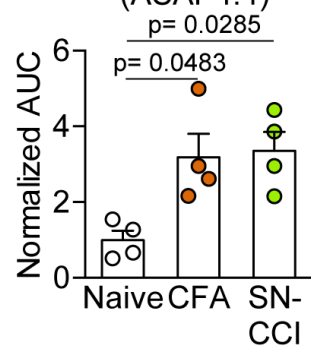

$2 \mathrm{~g}$ von Frey

(ASAP4.4)

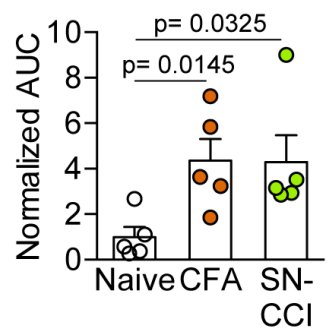

Figure 2

In vivo optical recording of mechanically (brush or von Frey)-induced neuronal activity in intact DRG neurons. a Optical voltage recordings of primary sensory neurons in response to the indicated stimuli in naïve, CFA, or SN-CCI animals. Purple bars indicate the timing of the stimulus application. Inset, images of DRG cell bodies expressing ASAP4.4. Yellow lines indicate $1.1 \mathrm{kHz}$ line scan regions where ASAP4.4 fluorescent signals were acquired. Scale bar: $5 \mu \mathrm{m}$. b In vivo DRG Ca2+ imaging from naïve or CFA-treated Pirt-GCaMP3 mice. (left) Averaged images before brush stimulus was applied. (right) Averaged images after brush stimulus was applied. White arrowheads indicate spontaneously activated neurons without application of stimulus. Yellow arrowheads indicate DRG neurons activated by brush stimulus. Scale bar: $100 \mu \mathrm{m}$. c GCaMP3 Ca2+ transients from individual DRG neurons (grey traces) and averaged Ca2+ transients from naive or CFA animals ( $n=3$ mice/treatment). Red arrow marks single action potential and 
red box indicates action potential burst. $d$ Mean area under the curve (AUC) of ASAP4.4 signals ( $n=3$ mice/group; Kruskal-Wallis test) and GCaMP3 Ca2+ transients ( $\mathrm{n}=3$ mice/group; two-tailed unpaired Student's t-test) in stimulated L5 DRG neurons. Each dot represents one neuron.

\section{Figure 3}

a

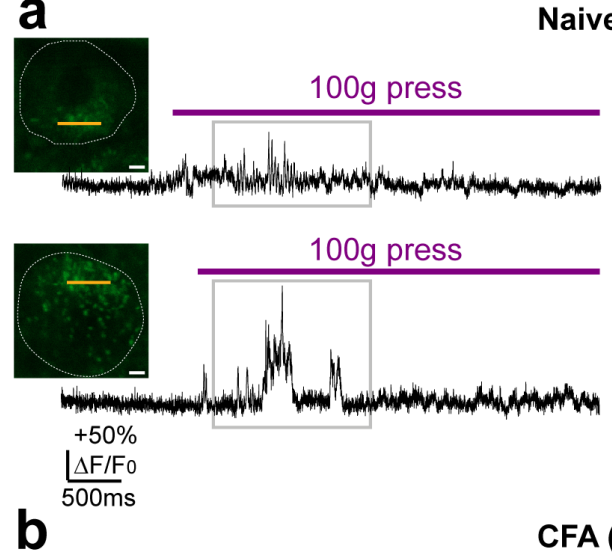

$-$


Baseline $100 \mathrm{~g}$ press
Naive (ASAP4.4)

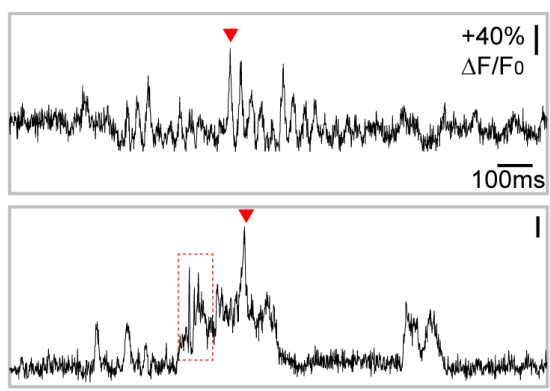

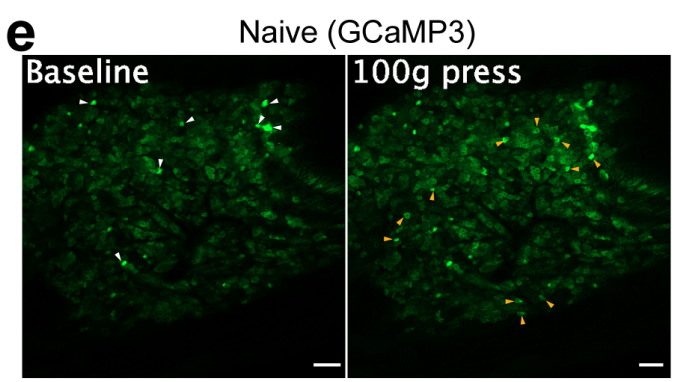

CFA (GCaMP3)

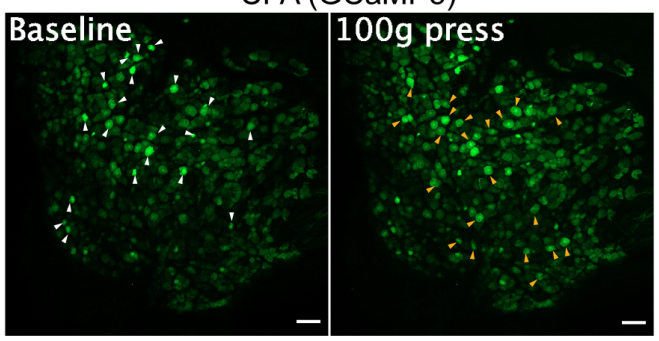

f

Naive
(GCaMP3)

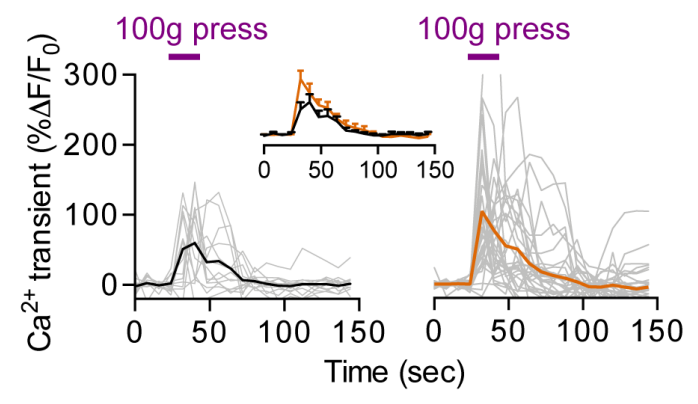

$9 \quad \begin{gathered}\text { Naive } \\ \text { (GCaMP3) }\end{gathered}$

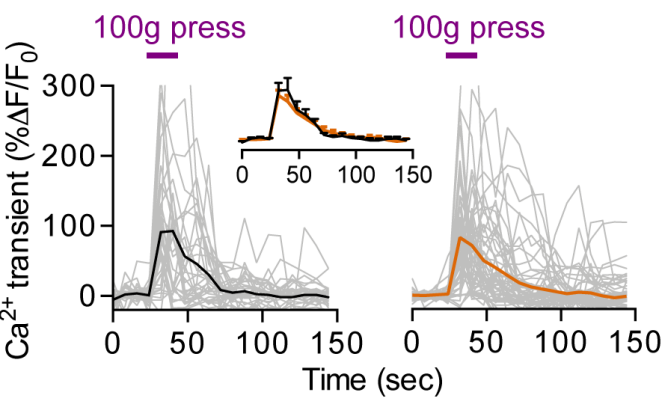

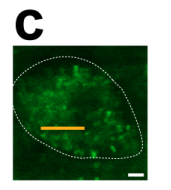

100g press

SN-CCI (ASAP4.4)
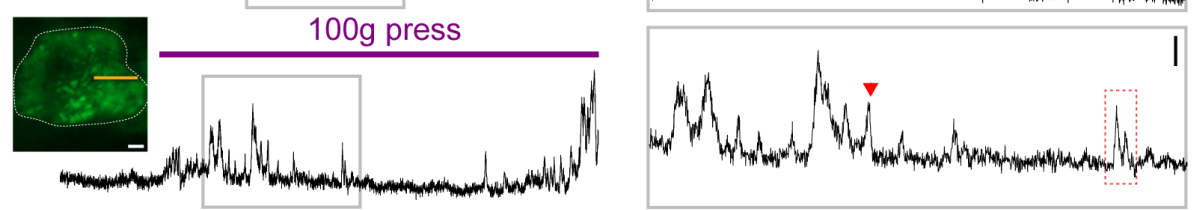

d

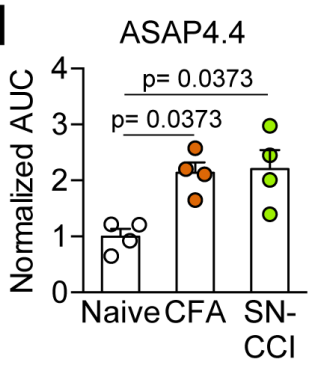

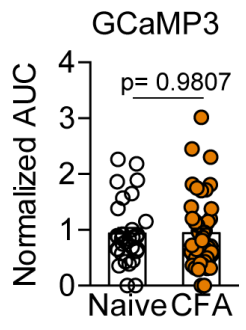

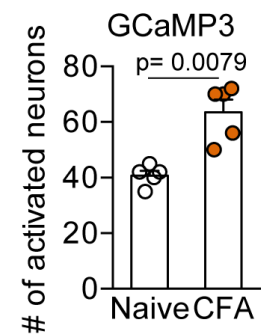

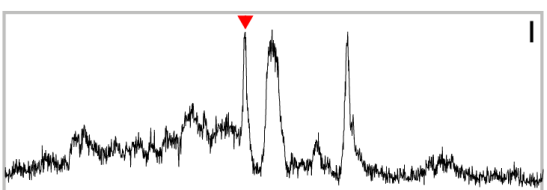

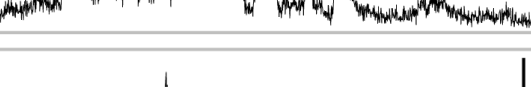

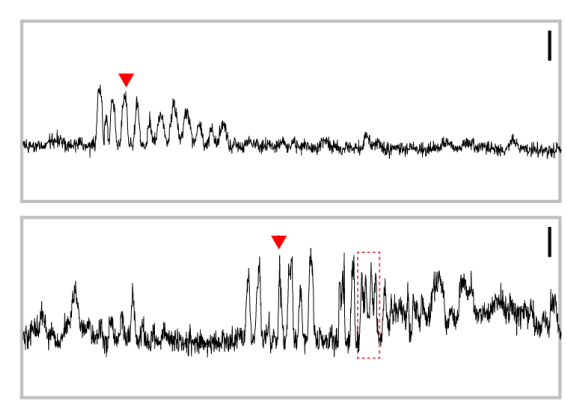

\section{Figure 3}

In vivo optical recording of mild press $(100 \mathrm{~g})$-induced neuronal activity in intact DRG neurons. a-c Optical voltage recordings of primary sensory neurons in response to a single mechanical force $(100 \mathrm{~g})$ 
applied to the hindpaw of naïve (a), CFA (b), or SN-CCI (c) animals. Insets, images of DRG cell bodies expressing ASAP4.4. Yellow lines indicate $1.1 \mathrm{kHz}$ line scan regions where ASAP4.4 optical recording signals were acquired. Each trace is the response of a single DRG neuron; (right) expanded view of the boxed region. Purple bars indicate the timing of the stimulus application. Representative line scan images in (b) are shown under the trace. Red arrow marks single action potential and red box indicates action potential burst. Scale bar: $5 \mu \mathrm{m}$. d Mean area under the curve (AUC) of ASAP4.4 signals (each dot represents one neuron, $n=4$ mice/treatment; Kruskal-Wallis test) and GCaMP3 Ca2+ transients (each dot represents one neuron, $n=3$ mice/treatment; Mann-Whitney U-test) in L5 DRG neurons in response to 100 $\mathrm{g}$ press, and the number of total activated neurons (each dot represents one animal, $n=5$ mice/treatment; Mann-Whitney U-test). e In vivo DRG Ca2+ imaging from naïve or CFA-treated Pirt-GCaMP3 mice. (left) Averaged images before application of mild press (100 g). (right) Averaged images after application of mild press $(100 \mathrm{~g})$. White arrowheads indicate spontaneously activated neurons in the absence of applied stimulus. Yellow arrowheads indicate DRG neurons activated by mild press. Scale bar: $100 \mu \mathrm{m} . \mathrm{f}-\mathrm{g}$ Ca2+ transients from DRG neurons in response to mild press $(100 \mathrm{~g})$ applied to the hindpaw using PirtGCaMP3 mice (grey), from one (f) or three (g) animals for each treatment. Traces of averaged Ca2+ transients from each group are shown in black or orange. 
Figure 4

a

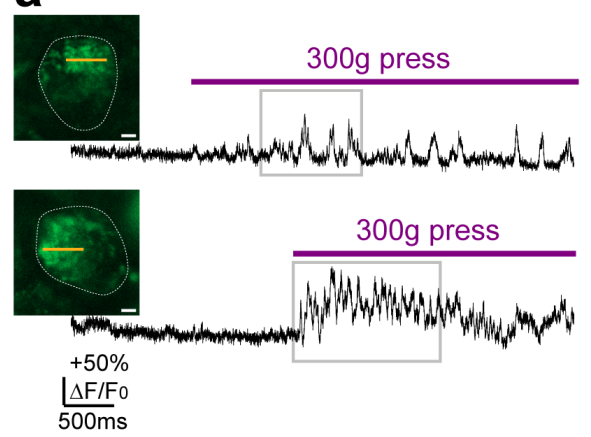

b

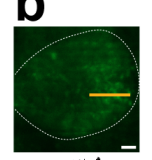

$300 \mathrm{~g}$ press

CFA (ASAP4.4)
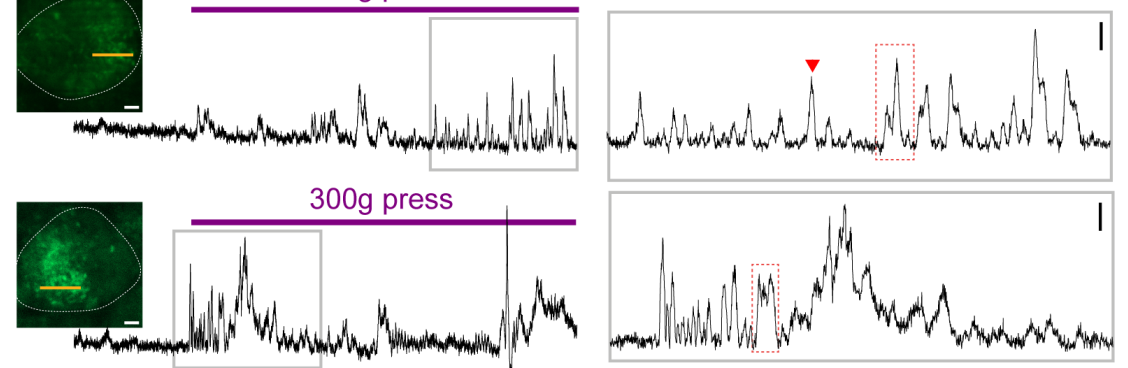

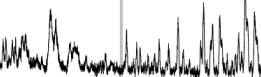

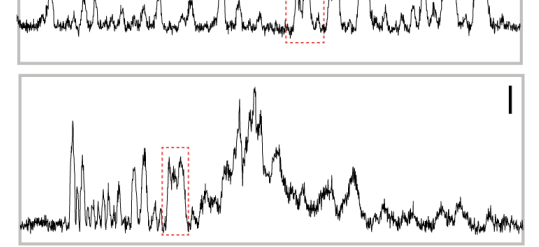

C

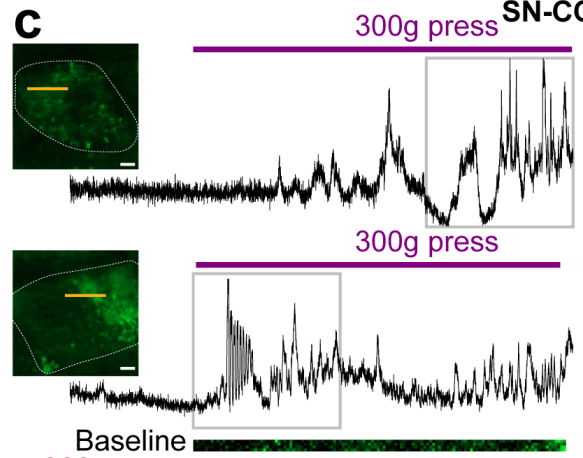

Baseline

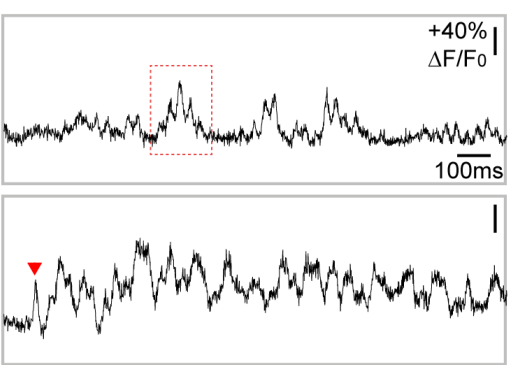

N-CCI (ASAP4.4)

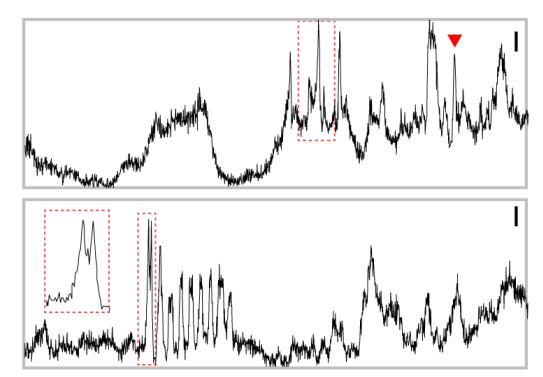

d
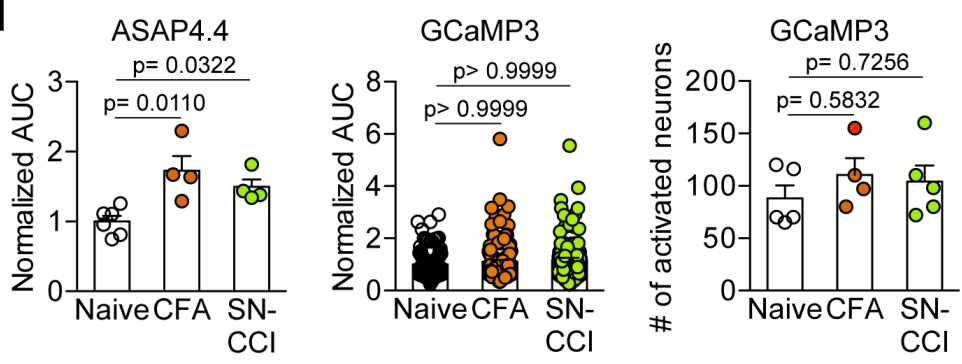

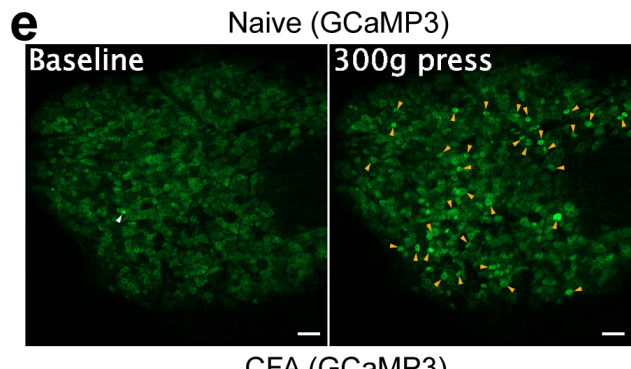

CFA (GCaMP3)

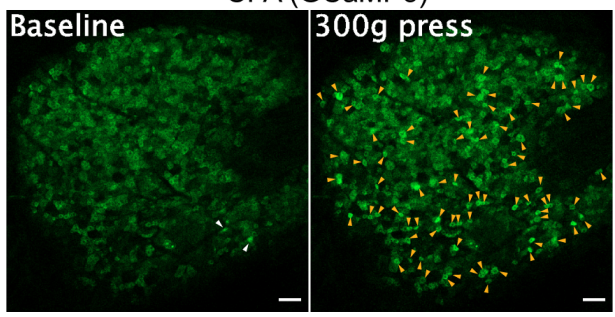

SN-CCI (GCaMP3)

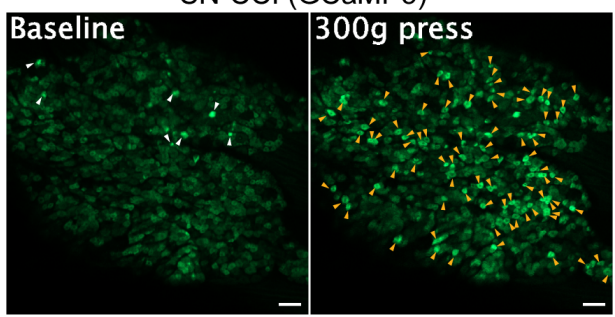

f $\quad \begin{gathered}\text { Naive } \\ \text { (GCaMP3 }\end{gathered}$

CFA

(GCaMP3)

С̊ 300g press

$300 \mathrm{~g}$ press

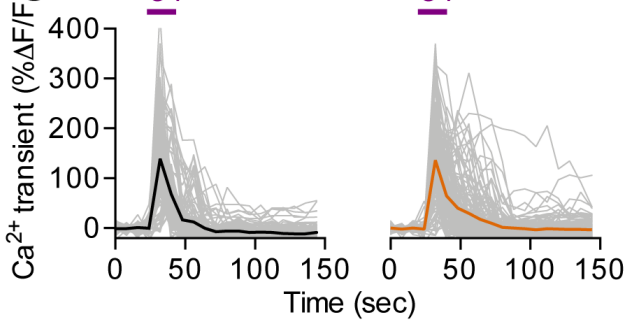

$\mathrm{SN}-\mathrm{CCl}$

(GCaMP3)

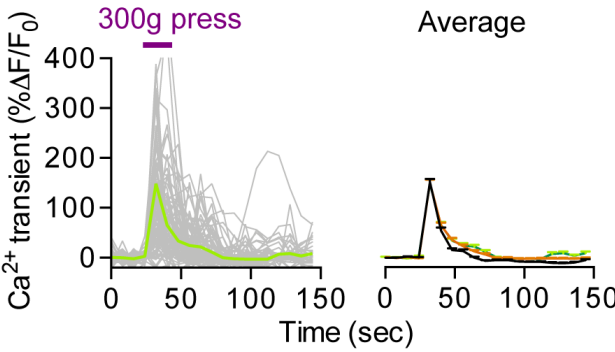

Figure 4

In vivo optical recording of strong press (300 g)-induced neuronal activity in intact DRG neurons. a-c Optical voltage recordings of primary sensory neurons in response to application of a single mechanical force (300 g) to the hindpaw of naïve (a), CFA (b), or SN-CCI (c) animals. Insets, images of DRG cell bodies expressing ASAP4.4. Yellow lines indicate $1.1 \mathrm{kHz}$ line scan regions where ASAP4.4 optical recording signals were acquired. Each trace is the response of a single DRG neuron; (right) expanded view 
of the boxed region shown on the right. Purple bars indicate the timing of the stimulus application. Representative line scan images in (c) are shown under the trace. Red arrow marks single action potentials and red box indicates action potential bursts. Scale bar: $5 \mu \mathrm{m}$. d Mean area under the curve (AUC) of ASAP4.4 signals (each dot represents one neuron, $n=4$ mice/treatment; Kruskal-Wallis test) and GCaMP3 Ca2+ transients (each dot represents one neuron, $\mathrm{n}=3$ mice/treatment; Kruskal-Wallis test) in L5 DRG neurons in response to $300 \mathrm{~g}$ press, and the number of total activated neurons (each dot represents one animal, $n=5$ mice/treatment; Kruskal-Wallis test). e In vivo DRG Ca2+ imaging from naïve, CFA, or SN$\mathrm{CCl}$ using Pirt-GCaMP3 mice. (left) Averaged images before application of strong press (300 g). (right) Averaged images after application of strong press $(300 \mathrm{~g})$. White arrowheads indicate spontaneously activated neurons in the absence of applied stimulus. Yellow arrowheads indicate DRG neurons activated by strong press. Scale bar: $100 \mu \mathrm{m}$. $\mathrm{f} \mathrm{Ca2}+$ transients from DRG neurons in response to strong press (300 g) applied to the hindpaw of Pirt-GCaMP3 mice (grey) from all naïve, CFA, or SN-CCl animals. Traces of averaged $\mathrm{Ca} 2+$ transients from each group are shown in black, orange, or green. 


\section{a}

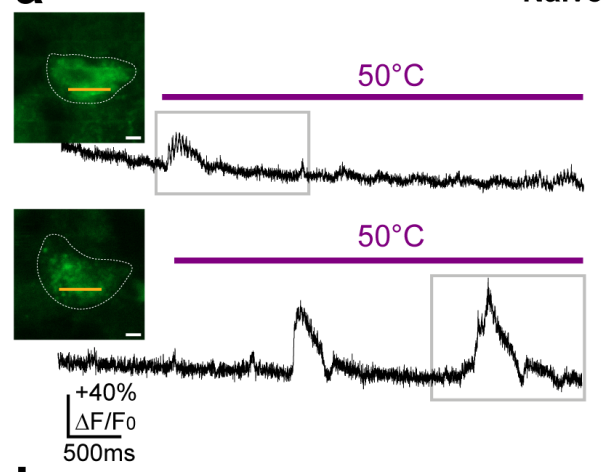

CFA (ASAP4.4)
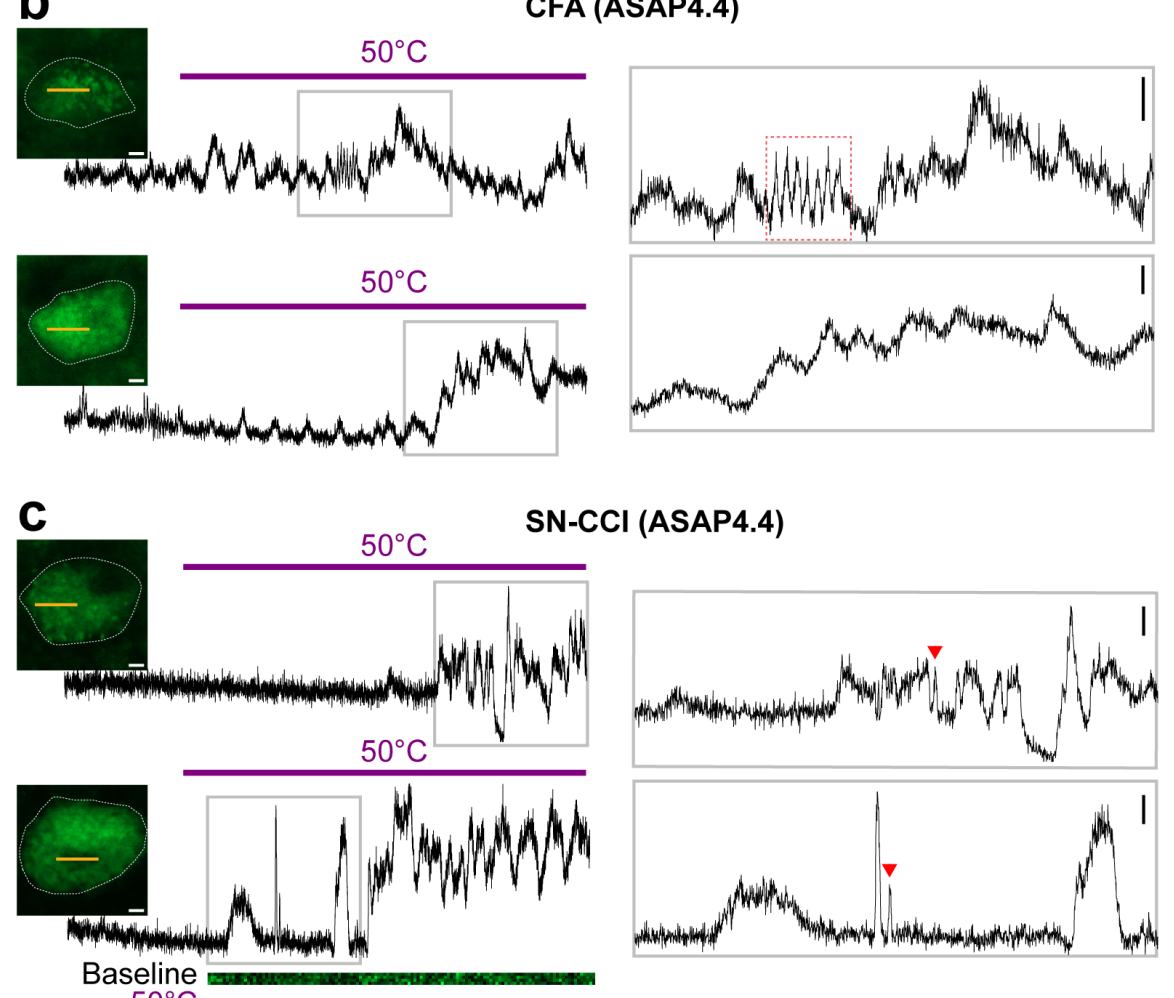

I (ASAP4.4)
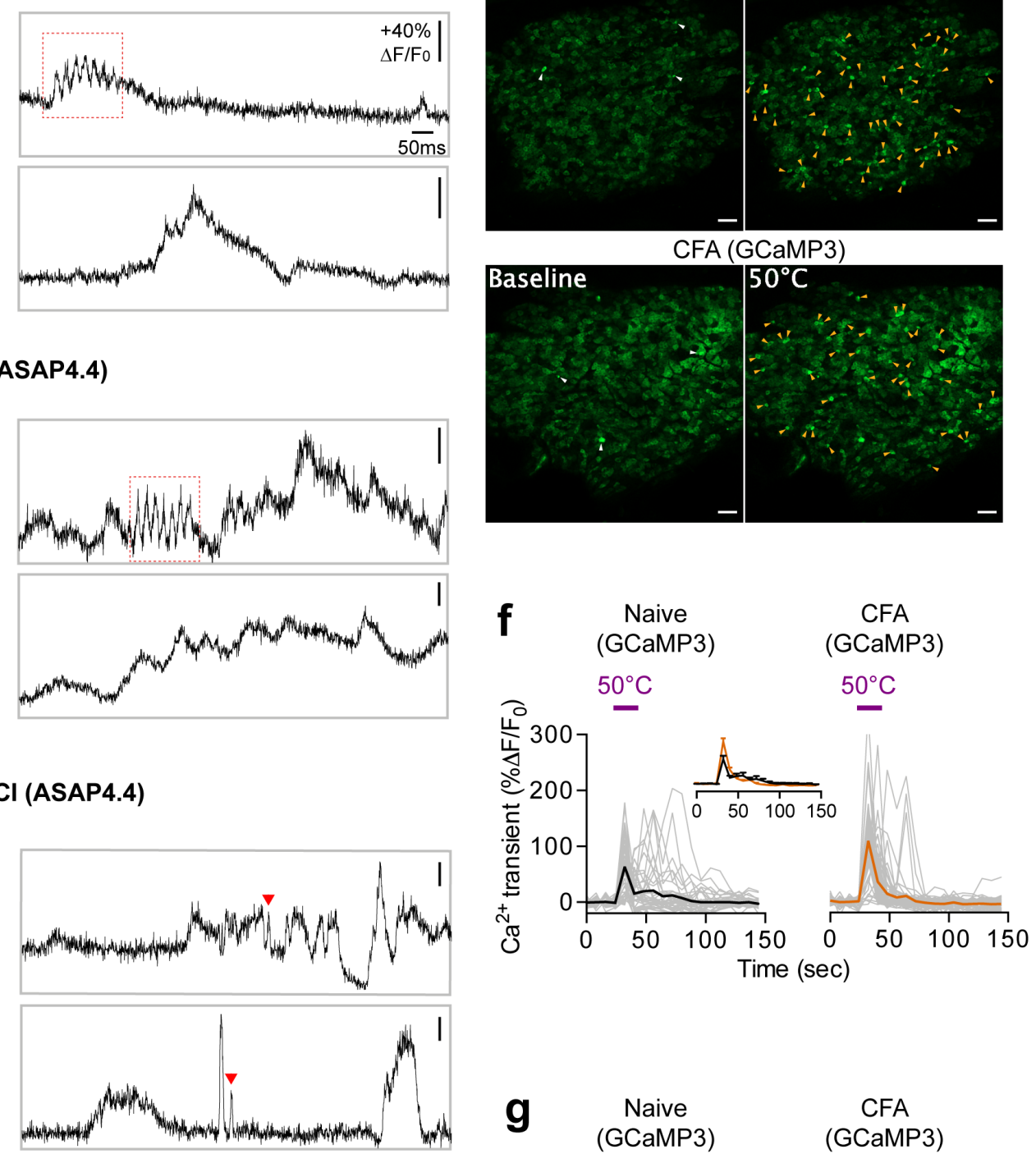

$50^{\circ} \mathrm{C}$

d
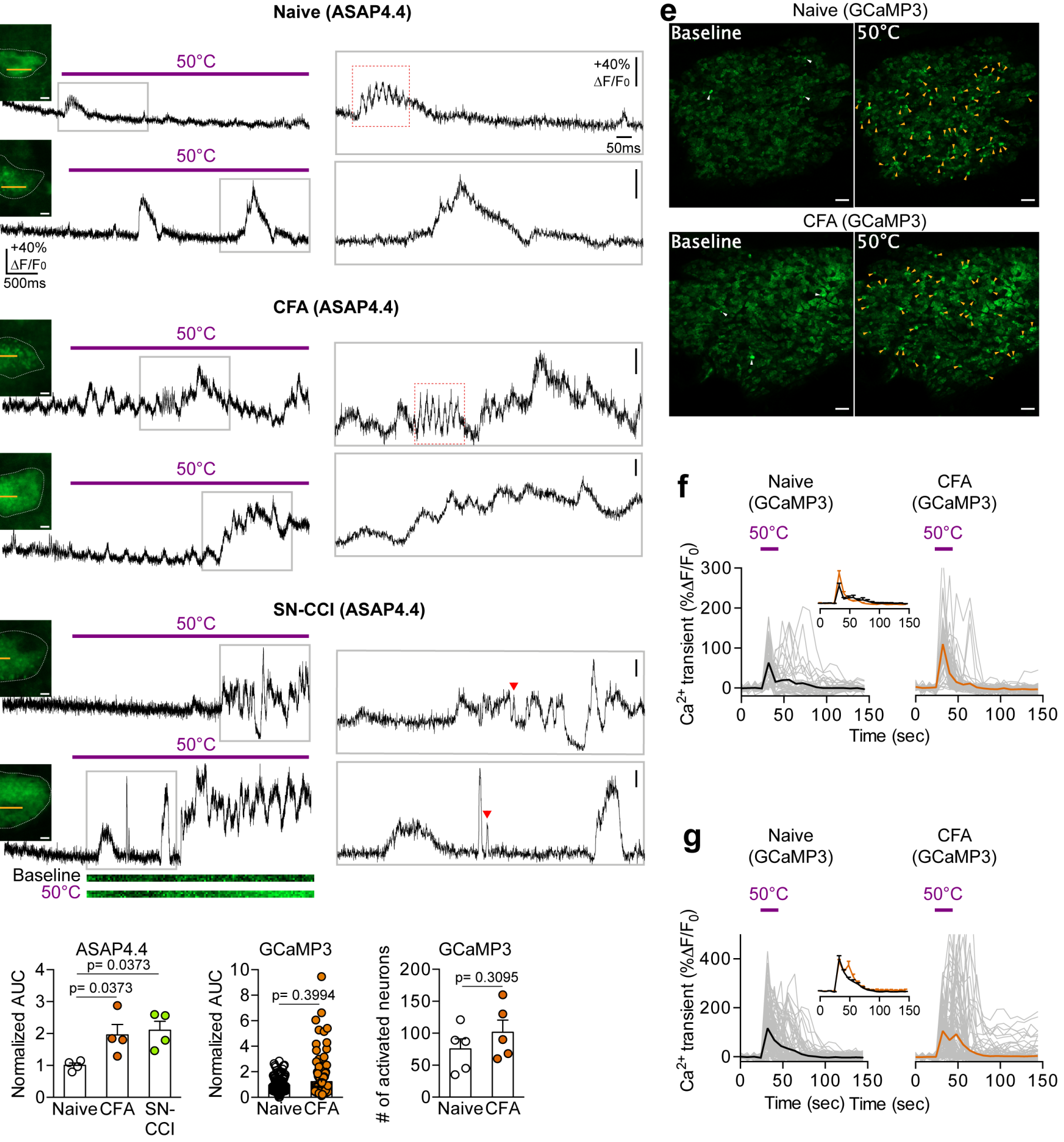

Figure 5

In vivo optical recording of neuronal activity in thermoreceptive neurons. a-c Optical voltage recordings of noxious heat $\left(50^{\circ} \mathrm{C}\right)$-sensitive primary sensory neurons from naïve (a), CFA (b), or SN-CCI (c) animals. Insets, images of DRG cell bodies expressing ASAP4.4. Yellow lines indicate $1.1 \mathrm{kHz}$ line scan regions where ASAP4.4 optical recording signals were acquired. Each trace is the response of a single DRG neuron; (right) expanded view of the boxed region. Purple bars indicate the timing of the stimulus 
application. Representative line scan images in (c) are shown under the trace. Red arrow marks single action potentials and red box indicates action potential bursts. Scale bar: $5 \mu \mathrm{m}$. $\mathrm{d}$ Mean area under the curve (AUC) of ASAP4.4 signals (each dot represents one neuron, $n=4$ mice/treatment; Kruskal-Wallis test) and GCaMP3 Ca2+ transients (each dot represents one neuron, $\mathrm{n}=3$ mice/treatment; Mann-Whitney U-test) in L5 DRG neurons in response to noxious heat stimulus, and the number of total activated neurons (each dot represents one animal, $n=5$ mice/treatment; Mann-Whitney U-test). e In vivo DRG Ca2+ imaging from naïve or CFA-treated Pirt-GCaMP3 mice. (left) Averaged images before application of heat stimulus $\left(50^{\circ} \mathrm{C}\right)$. (right) Averaged images after application of heat stimulus $\left(50^{\circ} \mathrm{C}\right)$. White arrowheads indicate spontaneously activated neurons in the absence of applied stimulus. Yellow arrowheads indicate DRG neurons activated by heat $\left(50^{\circ} \mathrm{C}\right)$. Scale bar: $100 \mu \mathrm{m} . \mathrm{f}-\mathrm{g} \mathrm{Ca} 2+$ transients from DRG neurons in response to heat $\left(50^{\circ} \mathrm{C}\right)$ applied to the hindpaw of Pirt-GCaMP3 mice (grey), from one (f) or three $(\mathrm{g})$ animals for each treatment. Traces of averaged $\mathrm{Ca} 2+$ transients of each group are shown in black or orange. 
a

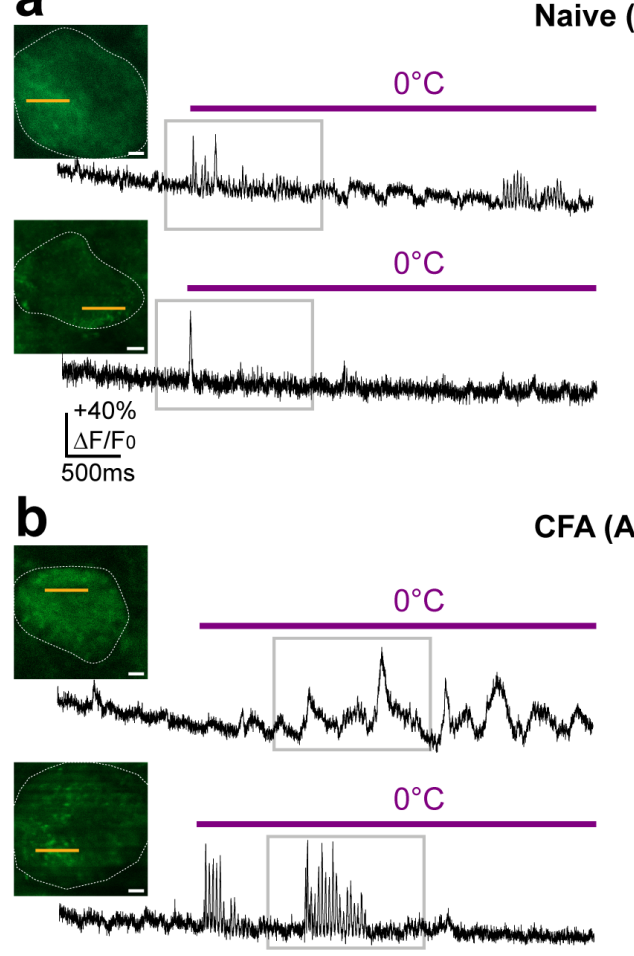

Baseline

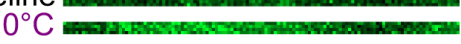

C

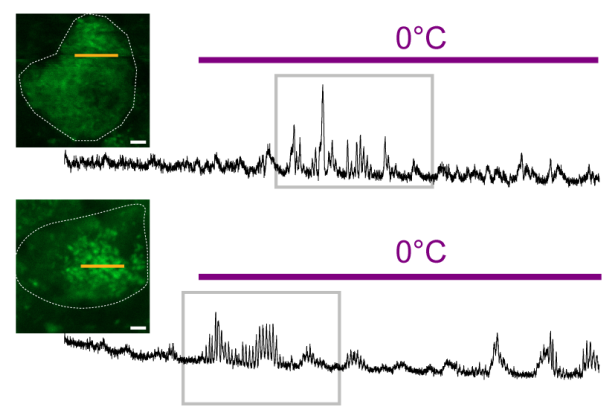

SN-CCI (ASAP4.4)
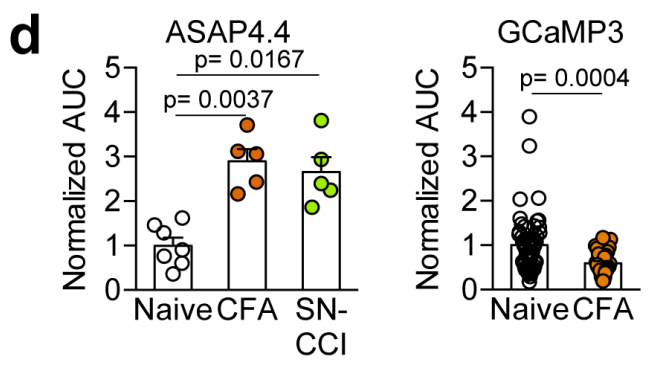
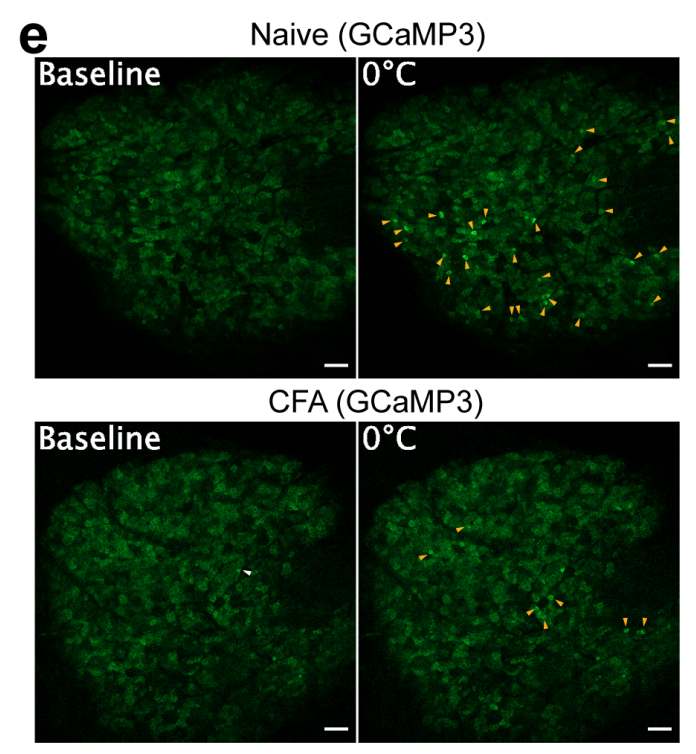

f $\quad \begin{gathered}\text { Naive } \\ \text { (GCaMP3) }\end{gathered}$

CFA

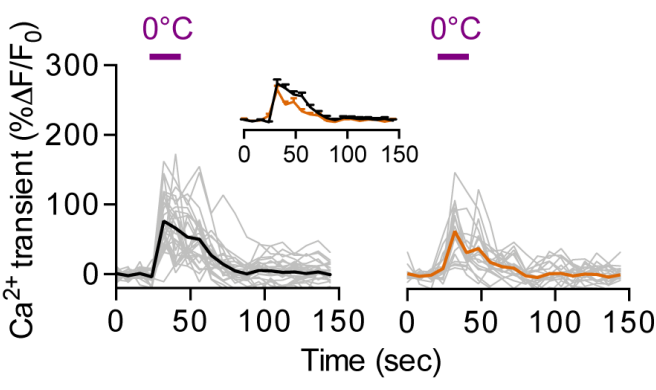

g $\quad \begin{gathered}\text { Naive } \\ \text { (GCaMP3) }\end{gathered}$

CFA

(GCaMP3)

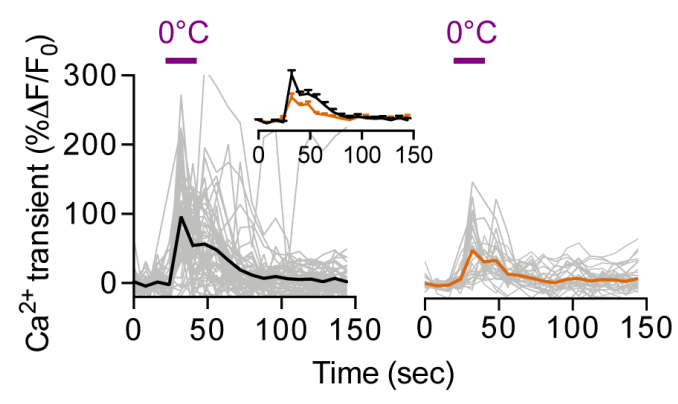

\section{Figure 6}

In vivo optical recording of neuronal activity in cold-sensing neurons. a-c Optical voltage recordings of cold $\left(0^{\circ} \mathrm{C}\right)$-sensitive primary sensory neurons from naïve (a), CFA (b), or SN-CCI (c) animals. Insets, images of DRG cell bodies expressing ASAP4.4. Yellow lines indicate $1.1 \mathrm{kHz}$ line scan regions where ASAP4.4 optical recording signals were acquired. Each trace is the response of a single DRG neuron; (right) expanded view of the boxed region. Purple bars indicate the timing of the stimulus application. 
Representative line scan images in (B) are shown under the trace. Red arrow marks single action potentials and red box indicates action potential bursts. Scale bar: $5 \mu \mathrm{m}$. d Mean area under the curve (AUC) of ASAP4.4 signals (each dot represents one neuron, $n=5$ mice/treatment; Kruskal-Wallis test) and GCaMP3 Ca2+ transients (each dot represents one neuron, $n=3$ mice/treatment; Mann-Whitney U-test) in L5 DRG neurons in response to noxious cold stimulus, and the number of total activated neurons (each dot represents one animal, $n=5$ mice/treatment; Mann-Whitney U-test). e In vivo DRG $\mathrm{Ca} 2+$ imaging from naïve or CFA-treated Pirt-GCaMP3 mice. (left) Averaged images before application of cold stimulus $\left(0^{\circ} \mathrm{C}\right)$. (right) Averaged images after application of cold stimulus $\left(0^{\circ} \mathrm{C}\right)$. White arrowhead indicate spontaneously activated neurons without application of stimulus. Yellow arrowheads indicate DRG neurons activated by cold $\left(0^{\circ} \mathrm{C}\right)$. Scale bar: $100 \mu \mathrm{m} . \mathrm{f}-\mathrm{g} \mathrm{Ca} 2+$ transients from DRG neurons in response to application of cold stimulus $\left(0^{\circ} \mathrm{C}\right)$ to the hindpaw of Pirt-GCaMP3 mice (grey), from one (f) or three (g) animals for each treatment. Traces of averaged $\mathrm{Ca} 2+$ transients from each group are shown in black or orange. 
Figure 7

a

Baseline

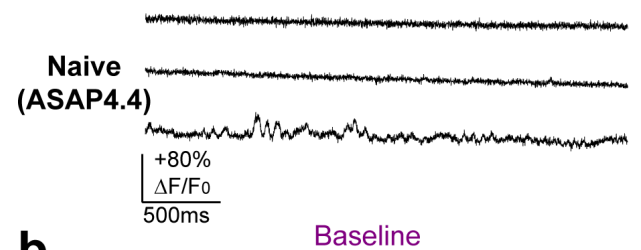

b

CFA

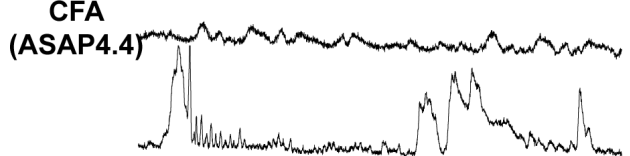

C

Baseline
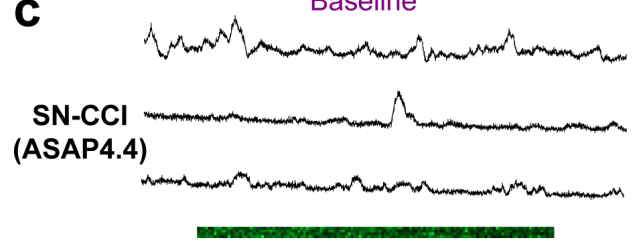

d $\begin{gathered}\text { Naive } \\ \text { (ASAP4.4) }\end{gathered}$

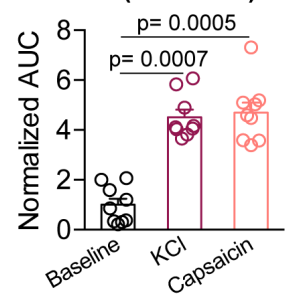

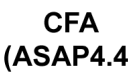

$p=0.0089$ $\mathrm{p}=\frac{0.0027}{\mathrm{O}_{\mathrm{O}}} \circ \mathrm{\circ}$

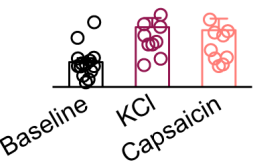

f
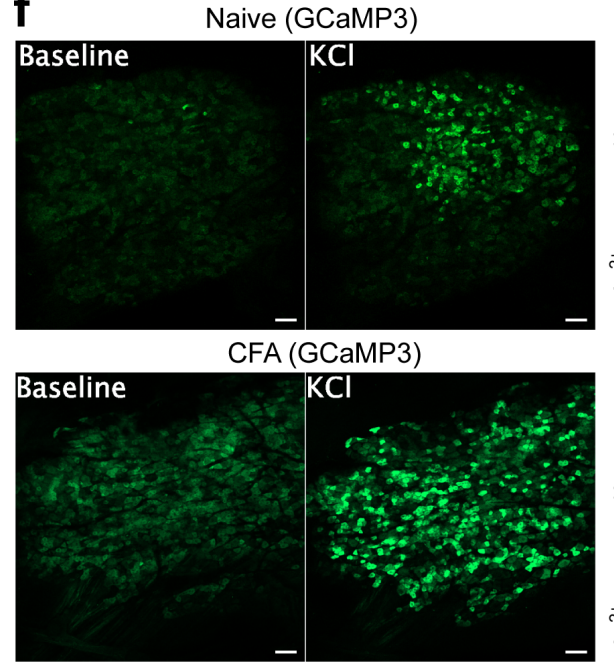
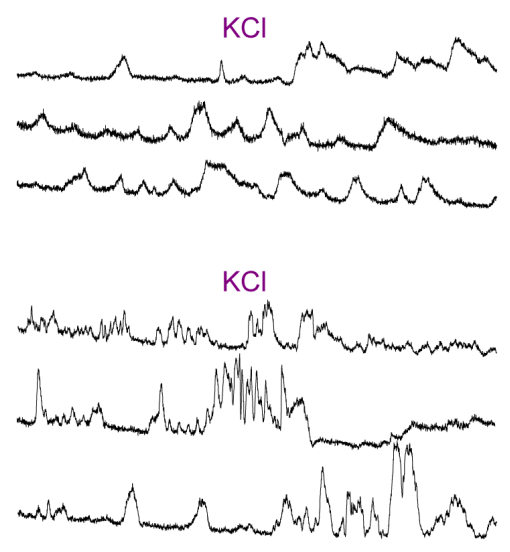

$\mathrm{KCl}$
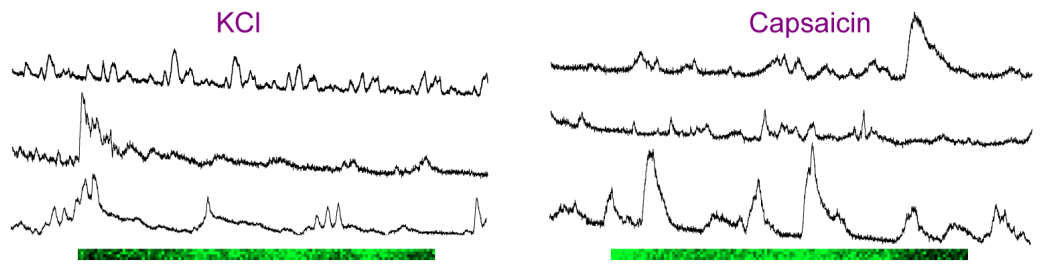

SN-CCl
(ASAP4.4)

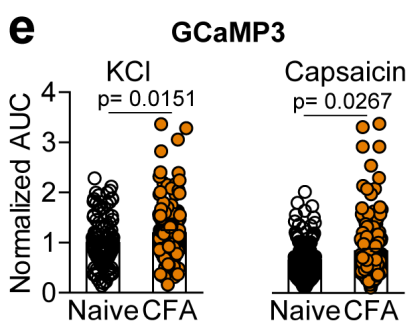

Naive (GCaMP3)
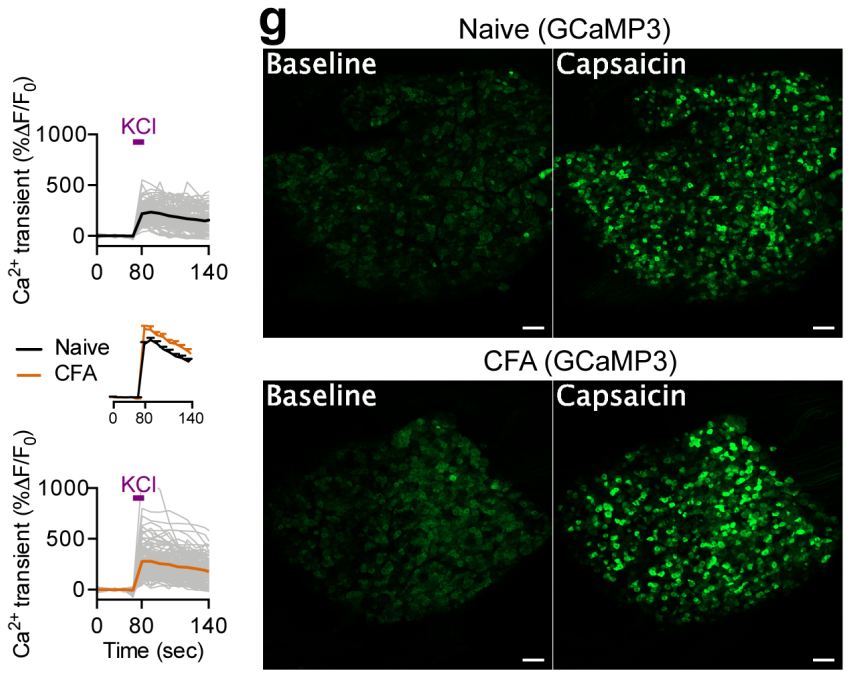

CFA (GCaMP3)

Capsaicin

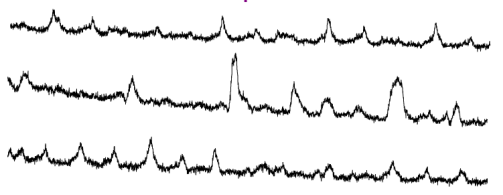

Capsaicin

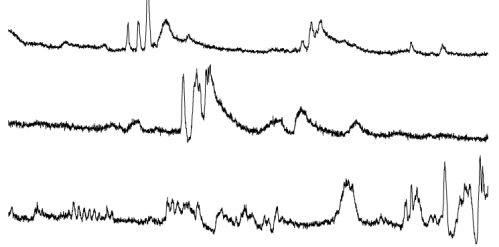

Capsaicin

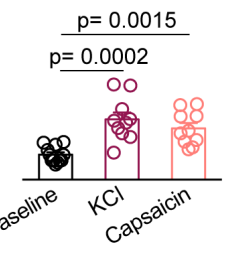

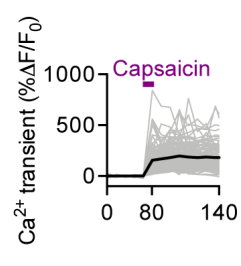

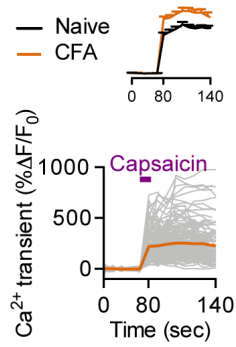

\section{Figure 7}

In vivo optical recording of intact DRG neurons in response to application of chemical stimuli (high potassium or capsaicin). a-c Representative traces of optical voltage recordings of primary sensory neurons before (baseline) and after topical application of $\mathrm{KCl}(50 \mathrm{mM})$ or capsaicin $(10 \mu \mathrm{M})$ to L5 DRG from naïve (a), CFA (b), or SN-CCl (c) animals. Representative line scan images in (c) are shown under the traces. d-e Mean area under the curve (AUC) of ASAP4.4 signals (d) (each dot represents one neuron, 
$\mathrm{n}=2-3$ mice/treatment; Kruskal-Wallis test) and GCaMP3 Ca2+ transients (e) (each dot represents one neuron, $\mathrm{n}=2-3$ mice/treatment; Mann-Whitney U-test) in L5 DRG neurons in response to indicated chemical stimuli. $f-g$ In vivo DRG Ca2+ imaging from naïve or CFA-treated Pirt-GCaMP3 mice. (left) Averaged images before application of indicated chemical stimuli. (middle) Averaged images after application of indicated chemical stimuli. Neurons activated by $\mathrm{KCl}$ or capsaicin are highlighted by bright fluorescence signals. Scale bar: $100 \mu \mathrm{m}$. (right) Ca2+ transients (gray traces) from DRG neurons in response to topical application of $\mathrm{KCl}(\mathrm{f})$ or capsaicin (g) onto DRG neurons from naïve or CFA-treated Pirt-GCaMP3 mice. Traces of averaged Ca2+ transients from each group are shown in black or orange.

\section{Supplementary Files}

This is a list of supplementary files associated with this preprint. Click to download.

- FigureS2DRGculture1025.tif

- FigureS4mCyRFP1025.tif

- FigureS3GCaMP3SP1025.tif

- FigureS1wholeDRG1025.tif

- NCsupplementaryinformationASAP42021YK.docx 